\title{
AdS monopole black hole and phase transition
}

\author{
Shoichiro Miyashita ${ }^{*}$ and Kei-ichi Maeda ${ }^{\dagger}$ \\ Department of Physics, Waseda University, Okubo 3-4-1, Shinjuku, Tokyo 169-8555, Japan
}

(Received 2 November 2016; published 23 December 2016)

\begin{abstract}
We study the Einstein-SO(3)Yang-Mills-Higgs system with a negative cosmological constant and find the monopole black hole solutions as well as the trivial Reissner-Nordström black hole. We discuss thermodynamical stability of the monopole black hole in an isolated system. We expect a phase transition between those two black holes when the mass of a black hole increases or decreases. The type of phase transition depends on the cosmological constant $\Lambda$, as well as the vacuum expectation value $v$ and the coupling constant $\lambda$ of the Higgs field. Keeping $\lambda$ small, we find there are two critical values of the cosmological constant, $\Lambda_{\operatorname{cr}(1)}(v)$ and $\Lambda_{\operatorname{cr}(2)}(v)$, which depend on $v$. If $\Lambda_{\operatorname{cr}(1)}(v)<\Lambda(<0)$, we find the first order transition, whereas if $\Lambda_{\operatorname{cr}(2)}(v)<\Lambda<\Lambda_{\operatorname{cr}(1)}(v)$, the transition becomes second order. For the case of $\Lambda_{b}(v)<\Lambda<\Lambda_{(2)}(v)$, we again find the first order irreversible transition from the monopole black hole to the extreme Reissner-Nordström black hole. Beyond $\Lambda_{b}(v)$, no monopole black hole exists. We also discuss thermodynamical properties of the monopole black hole in a thermal bath system.
\end{abstract}

DOI: 10.1103/PhysRevD.94.124037

\section{INTRODUCTION}

The black hole $(\mathrm{BH})$ has been a central object in relativistic astrophysics, as well as in gravitational physics, since the discovery of the Schwarzschild solution. One of the most important findings is the uniqueness of the Kerr solution [1]. An axisymmetric stationary vacuum solution, which describes a rotating $\mathrm{BH}$, is uniquely determined by the Kerr solution. In the Einstein-Maxwell theory, it was also proven that a $\mathrm{BH}$ solution is uniquely given by the Kerr-Newman solution with its global charges. This is the so-called black hole no-hair theorem.

However, if we extend matter fields to a more general class, we find a different type of solution called a hairy BH. After Bartnik and McKinnon (BM) discovered a nontrivial soliton solution in the Einstein-Yang-Mills (YM) theory, the colored $\mathrm{BH}$ was found in the same system [2,3]. This hairy $\mathrm{BH}$ has the same global charges as the Schwarzschild $\mathrm{BH}$. Hence, the uniqueness of a black hole solution is no longer applied when we consider a YM field. A YM hair, which is not a global charge, appears for such a $\mathrm{BH}$. Although it turns out that the colored $\mathrm{BH}$, as well as the BM soliton solution, is unstable, in the extended models, asymptotically flat non-Abelian BH solutions were intensively studied a few decades ago [4,5]. One of the most interesting non-Abelian $\mathrm{BHs}$ is a monopole $\mathrm{BH}$, which is obtained in the system with an $\mathrm{SU}(2)$ [SO(3)] Yang-Mills field and a real triplet Higgs field. Before the BM solution, the study of the Einstein-SO(3)Yang-Mills-Higgs (EYMH) system was performed from the viewpoint of a generalization of the 't Hooft-Polyakov monopole in a curved spacetime [6]. The asymptotically flat monopole $\mathrm{BH}$ was

\footnotetext{
*miyashita-at-gravity.phys.waseda.ac.jp

maeda-at-waseda.jp
}

found [7-9] soon after the discovery of a self-gravitating 't Hooft-Polyakov monopole, and the various properties were investigated [10-17]. The monopole $\mathrm{BH}$ is a magnetically charged $\mathrm{BH}$ with a global monopole charge, and its event horizon is located inside a gravitating monopole. One of the most important properties is its stability. In this system, there are two $\mathrm{BH}$ solutions, the monopole $\mathrm{BH}$ and the Reissner-Nordström ( $\mathrm{RN}) \mathrm{BH}$, with the same mass. The monopole $\mathrm{BH}$, if it exists, is dynamically stable against the perturbations [17], while the RN BH with the same horizon radius becomes unstable. However, since the event horizon of the monopole $\mathrm{BH}$ exists inside a monopole whose radius is determined by the coupling constants, when we increase the horizon radius, the nontrivial monopole structure outside the event horizon disappears, resulting in a unique stable RN BH. This behavior of stability is well understood through catastrophe theory [18].

This phenomenon is also easily reinterpreted via $\mathrm{BH}$ thermodynamics [19]. The laws of $\mathrm{BH}$ dynamics look similar to those of thermodynamics. This analogy leads to such real physical laws as $\mathrm{BH}$ thermodynamics after the discovery of Hawking radiation [20]. In BH thermodynamics, the Arnowitt-Deser-Misner (ADM) mass, the area of the event horizon, and the surface gravity correspond to the internal energy, the entropy, and the temperature, respectively. The other globally conserved quantities such as the electric (magnetic) charge or the angular momentum are also treated as thermodynamical quantities. Using thermodynamical variables such as BH entropy, we can discuss various types of phase transitions depending on the coupling constants and parameters.

In the $\mathrm{SO}(3)$ EYMH case, the monopole $\mathrm{BH}$ solution is found as a new phase possessing higher entropy than that of the RN BH. Hence, we expect that a phase transition 
between the monopole $\mathrm{BH}$ and the $\mathrm{RN} \mathrm{BH}$ occurs. Actually, the second order phase transition between these two BHs was found. However, in a certain parameter region, we also find the "reversible" first order phase transition between these two BHs, and the "nonreversible" first order phase transition from the monopole $\mathrm{BH}$ to the extreme RN BH [10].

Recently, in the context of the AdS/CFT correspondence [21], a black hole with asymptotically anti-de Sitter (AdS) spacetime has been attracting the most attention. Such a black hole has been studied intensively during the last decade [22]. A particlelike solution and a black hole in the EYM system with a negative cosmological constant were found in 1999 and 2000 [23,24]. It turns out that such solutions are stable. Their thermodynamical properties were also analyzed $[25,26]$.

Here, we study the EYMH system with a negative cosmological constant. A particlelike solution (a gravitating monopole) was found in $[27,28]$. We construct a magnetically charged $\mathrm{BH}$ solution and investigate its classical properties and thermodynamical properties. We examine the dependence of a negative cosmological constant on the phase transition. We should note that different spacetime solutions in the $\mathrm{SO}(3) \mathrm{EYMH}$ system were found, and some of them were applied to a holographic analysis for a strongly coupled gauge field [29-37].

The paper is organized as follows. We introduce the Einstein-Yang-Mills-Higgs system and provide the basic equations for a spherically symmetric static spacetime in Sec. II. In Sec. III, we present a monopole black hole as well as a self-gravitating monopole solution. We then analyze the thermodynamics of the present system in Sec. IV. We discuss the phase transition between the monopole $\mathrm{BH}$ and the $\mathrm{RN}$ $\mathrm{BH}$, whose order depends on a cosmological constant as well as the vacuum expectation value (VEV) of the Higgs field. We also show how the Hawking-Page transition occurs in the thermal equilibrium state. We summarize our results and give some remarks in Sec. V.

\section{EINSTEIN-YANG-MILLS-HIGGS SYSTEM WITH A COSMOLOGICAL CONSTANT}

\section{A. Basic equations}

We consider the EYMH system with a cosmological constant $\Lambda$ whose action is given by

$$
\begin{aligned}
I_{\mathrm{EH}} & =\int d^{4} x \sqrt{-g}\left[\mathcal{L}_{\mathrm{G}}+\mathcal{L}_{\mathrm{YM}}+\mathcal{L}_{\mathrm{H}}\right], \\
\mathcal{L}_{\mathrm{G}} & =\frac{1}{16 \pi G}(R-2 \Lambda), \\
\mathcal{L}_{\mathrm{YM}} & =-\frac{1}{4} F_{\mu \nu}^{(a)} F^{(a) \mu \nu}, \\
\mathcal{L}_{\mathrm{H}} & =-\frac{1}{2} D_{\mu} \Phi^{(a)} D^{\mu} \Phi^{(a)}-\frac{\lambda}{4}\left(\Phi^{(a)} \Phi^{(a)}-v^{2}\right)^{2},
\end{aligned}
$$

where $F_{\mu \nu}^{(a)}$ is an $\mathrm{SO}(3)$ YM field defined by

$$
F_{\mu \nu}^{(a)}=\partial_{\mu} A_{\nu}^{(a)}-\partial_{\nu} A_{\mu}^{(a)}-e \epsilon^{a b c} A_{\mu}^{(b)} A_{\nu}^{(c)},
$$

with $A_{\mu}^{(a)}$ and $e$ being the gauge field potential and the YM coupling constant. $\Phi^{(a)}, v$, and $\lambda$ are a real Higgs triplet, its VEV, and a self-coupling constant, respectively. ${ }^{1}$

The covariant derivative $D_{\mu}$ is defined by

$$
D_{\mu} \Phi^{(a)}=\partial_{\mu} \Phi^{(a)}-e \epsilon^{a b c} A_{\mu}^{(b)} \Phi^{(c)} .
$$

The Higgs mass $m_{\mathrm{H}}$, the gauge boson mass $m_{A}$, and their Compton wavelengths $\ell_{\mathrm{H}}$ and $\ell_{A}$ are given by

$$
\begin{aligned}
& m_{\mathrm{H}}=\sqrt{\lambda} v, \quad \ell_{\mathrm{H}}=\frac{1}{\sqrt{\lambda} v}, \\
& m_{A}=e v, \quad \ell_{A}=\frac{1}{e v} .
\end{aligned}
$$

We will normalize all variables by the Compton wavelength of the YM field, $\ell_{A}$, with the units $\hbar=c=1$. The Planck mass is defined by $M_{\mathrm{PL}}=G^{-1 / 2}$.

\section{B. A spherically symmetric static system}

In order to discuss a gravitating monopole and a monopole $\mathrm{BH}$, we consider a spherically symmetric static system and assume the hedgehog ansatz for the $\mathrm{SO}(3) \mathrm{YM}$ field and the Higgs field as

$$
\begin{aligned}
\Phi^{(a)} & =v \hat{r}^{(a)} h(r), \\
A_{i}^{(a)} & =w_{i}^{(c)} \epsilon^{a b c} \hat{r}^{(b)} \frac{1-w(r)}{e r},
\end{aligned}
$$

where $w_{i}^{(a)}$ is the triad of the 3 -space and $\hat{r}^{(a)}$ is the unit "radial" vector in the internal space.

The static and spherically symmetric metric is described as follows:

$$
d s^{2}=-f(r) e^{-2 \delta(r)} d t^{2}+f(r)^{-1} d r^{2}+r^{2} d \theta^{2}+r^{2} \sin ^{2} \theta d \phi^{2},
$$

where

$$
f(r)=1-\frac{2 G m(r)}{r}-\frac{\Lambda}{3} r^{2}
$$

and $\delta(r)$ are metric functions which depend only on the radial coordinate $r$.

\footnotetext{
${ }^{1}$ The Greek indices $(\mu, \nu, \cdots)$ and the Latin indices $(i, j, \cdots)$ run the spacetime coordinates $(0,1,2,3)$ and the spatial coordinates $(1,2,3)$, while the Latin indices with round brackets $[(a),(b), \cdots]$ describe the internal coordinates.
} 
Varying the action (2.1) with respect to the metric $g^{\mu \nu}$, the Higgs field $\Phi^{(a)}$, and the YM gauge field $A_{\mu}^{(a)}$, we find four basic equations.

Introducing the dimensionless variables $\tilde{r} \equiv r / \ell_{A}$, $\tilde{m} \equiv G m / \ell_{A}$ as well as the dimensionless parameters $\tilde{v} \equiv v / M_{\mathrm{PL}}, \quad \tilde{\Lambda} \equiv \Lambda \ell_{A}^{2}$, the four basic equations are written as

$$
\begin{aligned}
\frac{d \tilde{m}}{d \tilde{r}}= & 4 \pi \tilde{v}^{2}\left[f\left\{\left(\frac{d w}{d \tilde{r}}\right)^{2}+\frac{\tilde{r}^{2}}{2}\left(\frac{d h}{d \tilde{r}}\right)^{2}\right\}\right. \\
& \left.+\frac{\left(w^{2}-1\right)^{2}}{2 \tilde{r}^{2}}+w^{2} h^{2}\right]+\pi \bar{\lambda} \tilde{v}^{2} \tilde{r}^{2}\left(h^{2}-1\right)^{2}, \\
& \frac{d \delta}{d \tilde{r}}=-8 \pi \tilde{v}^{2} \tilde{r}\left[\frac{1}{\tilde{r}^{2}}\left(\frac{d w}{d \tilde{r}}\right)^{2}+\frac{1}{2}\left(\frac{d h}{d \tilde{r}}\right)^{2}\right],
\end{aligned}
$$$$
\frac{d^{2} w}{d \tilde{r}^{2}}=\frac{1}{\tilde{r}^{2} f}\left[w\left(w^{2}-1+\tilde{r}^{2} h^{2}\right)-2\left(\tilde{m}-\frac{\tilde{\Lambda}}{3} \tilde{r}^{3}\right) \frac{d w}{d \tilde{r}}\right.
$$$$
\left.+8 \pi \tilde{v}^{2} \tilde{r} \frac{d w}{d \tilde{r}}\left\{\frac{\left(w^{2}-1\right)^{2}}{2 \tilde{r}^{2}}+w^{2} h^{2}+\frac{\bar{\lambda}}{4} \tilde{r}^{2}\left(h^{2}-1\right)^{2}\right\}\right],
$$

$$
\begin{aligned}
\frac{d^{2} h}{d \tilde{r}^{2}}= & -\frac{2 d h}{\tilde{r}} \frac{d \tilde{r}}{d \tilde{r}}+\frac{1}{\tilde{r}^{2} f}\left[2 h w^{2}+\bar{\lambda} \tilde{r}^{2} h\left(h^{2}-1\right)-2\left(\tilde{m}-\frac{\tilde{\Lambda}}{3} \tilde{r}^{3}\right) \frac{d h}{d \tilde{r}}\right. \\
& \left.+8 \pi \tilde{v}^{2} \tilde{r} \frac{d h}{d \tilde{r}}\left\{\frac{\left(w^{2}-1\right)^{2}}{2 \tilde{r}^{2}}+w^{2} h^{2}+\frac{\bar{\lambda}}{4} \tilde{r}^{2}\left(h^{2}-1\right)^{2}\right\}\right],
\end{aligned}
$$

where we define $\bar{\lambda}=\lambda / e^{2}$.

\section{Boundary conditions}

Next, we consider the boundary conditions.

\section{Boundary conditions at a center or at a horizon}

For a gravitating monopole, we impose a regularity at the center $(\tilde{r}=0)$. We find

$$
\begin{gathered}
w(\tilde{r})=1-c_{w} \tilde{r}^{2}+o\left(\tilde{r}^{3}\right), \\
h(\tilde{r})=c_{h} \tilde{r}+o\left(\tilde{r}^{2}\right), \\
\tilde{m}(\tilde{r})=c_{m} \tilde{r}^{3}+o\left(\tilde{r}^{4}\right),
\end{gathered}
$$

with

$$
c_{m}=\frac{4 \pi}{3} \tilde{v}^{2}\left(6 c_{w}^{2}+\frac{3}{2} c_{h}^{2}+\frac{1}{4} \bar{\lambda}\right) .
$$

The constants $c_{w}$ and $c_{h}$ are the shooting parameters, which are fixed by the asymptotically AdS condition.

For a black hole solution, we impose a regularity at the horizon $\tilde{r}=\tilde{r}_{H}$, which is defined by $f\left(\tilde{r}_{H}\right)=0$, i.e.,

$$
\tilde{m}\left(\tilde{r}_{H}\right)=\frac{\tilde{r}_{H}}{2}\left(1-\frac{\tilde{\Lambda}}{3} \tilde{r}_{H}^{2}\right) .
$$

We then find that the first derivatives of $w(\tilde{r})$ and $h(\tilde{r})$ on the horizon are given as

$$
\begin{aligned}
& \left.\frac{d w}{d \tilde{r}}\right|_{\tilde{r}=\tilde{r}_{H}}=\frac{\tilde{r}_{H} w_{H}\left(w_{H}^{2}-1+\tilde{r}_{H}^{2} h_{H}^{2}\right)}{\left(1-\tilde{\Lambda} \tilde{r}_{H}^{2}\right)-2 \pi \tilde{v}^{2}\left[2\left(w_{H}^{2}-1\right)^{2}+4 \tilde{r}_{H}^{2} w_{H}^{2} h_{H}^{2}+\bar{\lambda} \tilde{r}_{H}^{4}\left(h_{H}^{2}-1\right)^{2}\right]}, \\
& \left.\frac{d h}{d \tilde{r}}\right|_{\tilde{r}=\tilde{r}_{H}}=\frac{\tilde{r}_{H} h_{H}\left(2 w_{H}^{2}+\bar{\lambda} \tilde{r}_{H}^{2}\left(h_{H}^{2}-1\right)\right)}{\left(1-\tilde{\Lambda} \tilde{r}_{H}^{2}\right)-2 \pi \tilde{v}^{2}\left[2\left(w_{H}^{2}-1\right)^{2}+4 \tilde{r}_{H}^{2} w_{H}^{2} h_{H}^{2}+\bar{\lambda} \tilde{r}_{H}^{4}\left(h_{H}^{2}-1\right)^{2}\right]},
\end{aligned}
$$

where $w_{H}=w\left(\tilde{r}_{H}\right)$ and $h_{H}=h\left(\tilde{r}_{H}\right)$. Giving the horizon radius $\tilde{r}_{H}$, we have to fix the remaining two constants $\left(w_{H}\right.$ and $h_{H}$ ) by the shooting method to find a regular BH with an asymptotically AdS spacetime. We assume that $f(\tilde{r})$ is always positive outside the horizon $\tilde{r}_{H}$ in order not to make an additional horizon.

\section{Boundary conditions at spatial infinity}

We assume that a gravitating monopole or a monopole black hole approaches an AdS spacetime at spatial infinity. Then we assume that the mass function $\tilde{m}$ is finite at infinity, i.e.,

$$
\tilde{m}(\infty)=\text { constant }
$$

which implies

$$
w(\infty)=0, \quad h(\infty)=1,
$$

as well as

$$
\delta(\infty)=0 .
$$

Under the above boundary conditions, we solve the basic equations. Before giving a nontrivial gravitating monopole 
or a monopole $\mathrm{BH}$ solution, we simply show a trivial $\mathrm{RN}$ $\mathrm{BH}$ solution as follows.

Trivial solution.-When we assume the variables as

$$
w(r)=0, \quad h(r)=1, \quad \text { and } \quad \delta(r)=0,
$$

Eqs. (2.9), (2.10), and (2.11) are trivially satisfied, and Eq. (2.8) becomes

$$
m^{\prime}(r)=\frac{2 \pi}{G e^{2} r^{2}}
$$

which is easily integrated as

$$
m(r)=M-\frac{2 \pi}{G e^{2} r}
$$

where

$$
M=\frac{r_{H}}{2 G}\left(1+\frac{4 \pi}{e^{2} r_{H}^{2}}-\frac{\Lambda}{3} r_{H}^{2}\right) .
$$

This is the Reissner-Nordström AdS BH solution with the ADM mass $M$ and the magnetic charge $2 \pi^{1 / 2} / e$.

\section{NONTRIVIAL SOLUTIONS}

As mentioned before, we have performed shooting for two parameters numerically in order to obtain nontrivial solutions, both a self-gravitating monopole and a monopole $\mathrm{BH}$. Although the monopole solution was obtained in $[27,28]$, we show both solutions below.

\section{A. Self-gravitating monopole}

We show some examples of gravitating monopole solutions in Fig. 1 for the case where $\bar{\lambda}=0.1$ and $\tilde{v}=0.1$. We choose $\tilde{\Lambda}=-1,-10,-20,-30$, and $-33.8 \approx \tilde{\Lambda}_{\mathrm{b}}$, beyond which there exists no monopole solution.

As $|\tilde{\Lambda}|$ increases, the monopole radius shrinks in the unit of $\ell_{A}=1$ and, as shown in Fig. 2, the minimum of the metric function $f(r)$ decreases and eventually vanishes at some radius $\left(r \approx 0.21 \ell_{A}\right)$ when the cosmological constant reaches the boundary value $\tilde{\Lambda}_{\mathrm{b}}(\tilde{v})$, which depends on $\tilde{v}^{2}$ This means that, if the typical monopole scale $\left(\sim \ell_{A}\right)$ is much larger than the AdS curvature length $\ell_{\Lambda}=\sqrt{-3 / \Lambda}$, no monopole solution is possible. Beyond the critical value, we find only the RN AdS BH.

We also show the monopole mass $M$ in terms of the cosmological constant $\tilde{\Lambda}$ in Fig. 3. The mass is determined

\footnotetext{
${ }^{2}$ The boundary value also depends on $\bar{\lambda}$, but we fix the value of $\bar{\lambda}$ in the text $(\bar{\lambda}=0.1)$ because it shows the most variety of phase behaviors. See Appendix A for details on its dependence for the case of $\tilde{\Lambda}=0$. We also find similar behavior for the case where $\tilde{\Lambda}<0$.
}
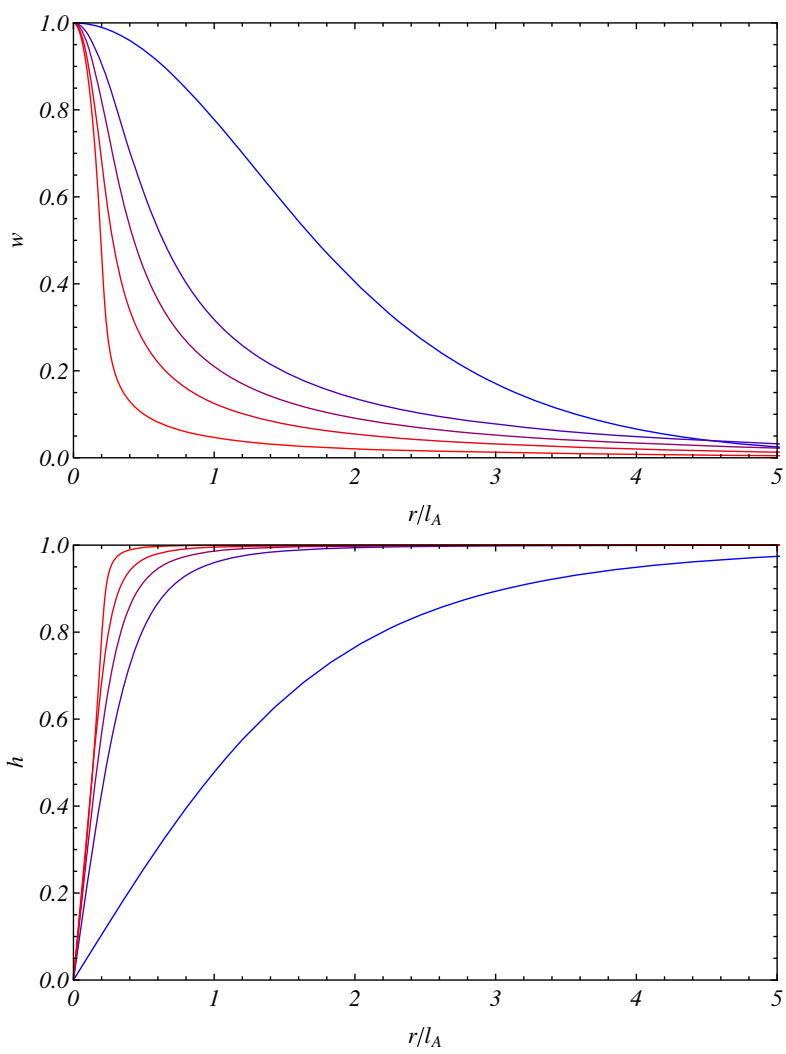

FIG. 1. A self-gravitating monopole. We show the Higgs field $h$ and the gauge potential $w$ in terms of the radius $\tilde{r}$. We set $\bar{\lambda}=0.1$ and $\quad \tilde{v}=0.1$. We choose $\tilde{\Lambda}=0,-10,-20,-30$ and $\tilde{\Lambda}=-33.8 \approx \tilde{\Lambda}_{\mathrm{b}}$, from the top curve to the bottom one in the case of $w$, and from the bottom one to the top one for $h$.

by the cosmological constant $\tilde{\Lambda}$ as well as the coupling constants $\bar{\lambda}$ and the VEV $\tilde{v}$. We confirm that the mass $M$ at the boundary value of the cosmological constant is the same as that of the extreme RN AdS BH.

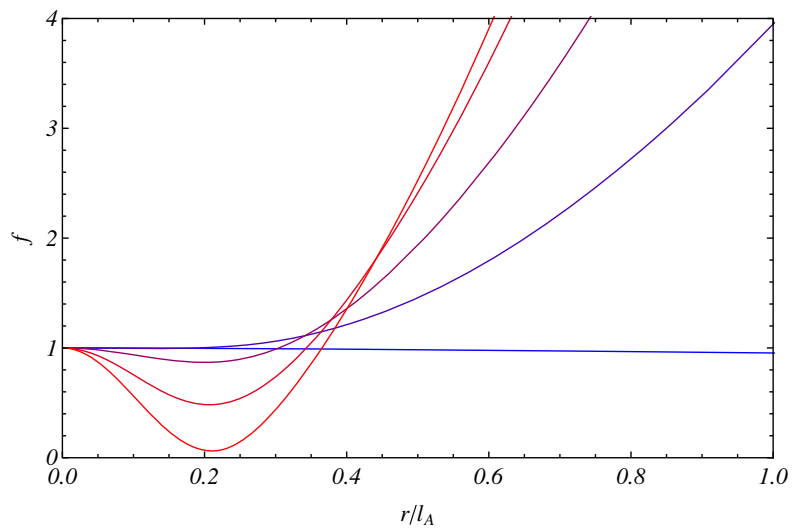

FIG. 2. The behavior of $f(\tilde{r})$ of the monopole solution, $\tilde{v}=0.1$ and $\bar{\lambda}=0.1$. The curves denote the cases of $\tilde{\Lambda}=0,-10,-20,-30$, and $-33.8 \approx \tilde{\Lambda}_{\mathrm{b}}$ from the top. 


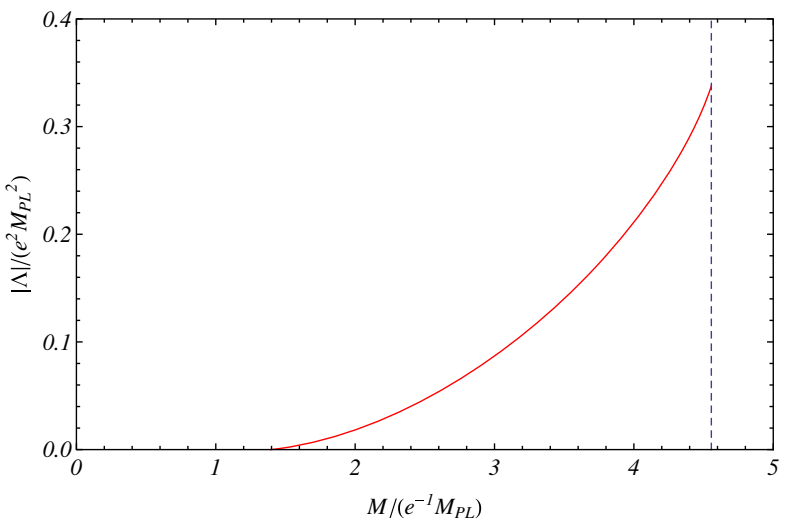

FIG. 3. The relation between the monopole mass $M$ and the cosmological constant $\Lambda$. The dashed line $\left(M \approx 4.55 M_{\mathrm{PL}} / e\right)$ represents the mass of extreme $\mathrm{RN}$ AdS BH when $\tilde{\Lambda}=-33.8 \approx \tilde{\Lambda}_{\mathrm{b}}$. We set $\bar{\lambda}=0.1$ and $\tilde{v}=0.1$.

We find similar behavior in the case of the asymptotically flat spacetime (see Appendix A). There exists a critical value of $\tilde{v}$, beyond which there is no monopole and the RN BH is obtained. When we include a cosmological constant, since the monopole structure is determined by $\tilde{\Lambda}$ as well as $\tilde{v}$, there is a boundary value of the cosmological constant,

$$
\tilde{\Lambda}=\tilde{\Lambda}_{\mathrm{b}}(\tilde{v}),
$$

beyond which no monopole solution can exist. We show this boundary curve in the $v$ - $\Lambda$ plane in Fig. 4 . We obtain the monopole solution as well as the RN AdS BH inside the boundary, while we find only the RN AdS BH solution outside of this boundary.

\section{B. Monopole black hole}

Now we present a monopole BH solution. In Fig. 5, we depict the gauge potential $w(\tilde{r})$ and the Higgs field $h(\tilde{r})$.

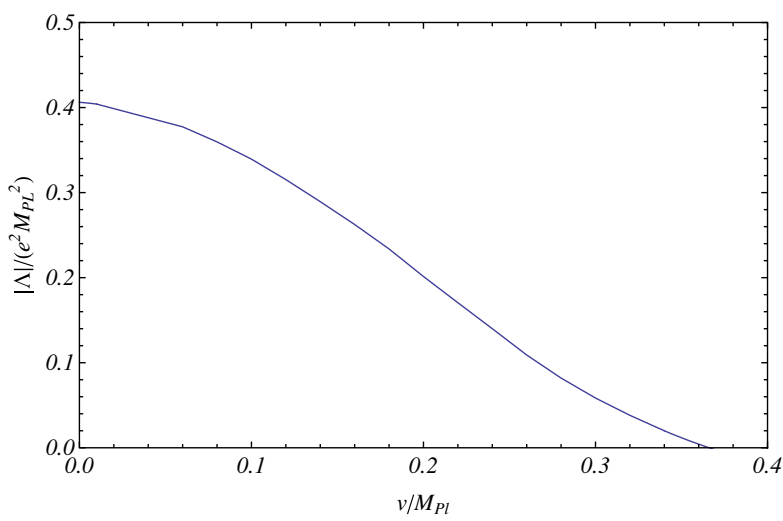

FIG. 4. The boundary value of the cosmological constant $\left[\Lambda=\Lambda_{\mathrm{b}}(v)\right]$ in the $v$ - $\Lambda$ plane, beyond which no monopole solution can exist. We set $\bar{\lambda}=0.1$.

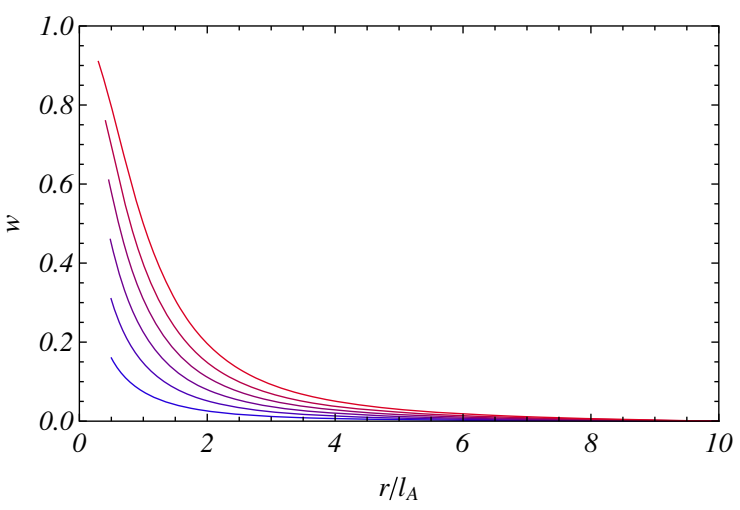

(a)

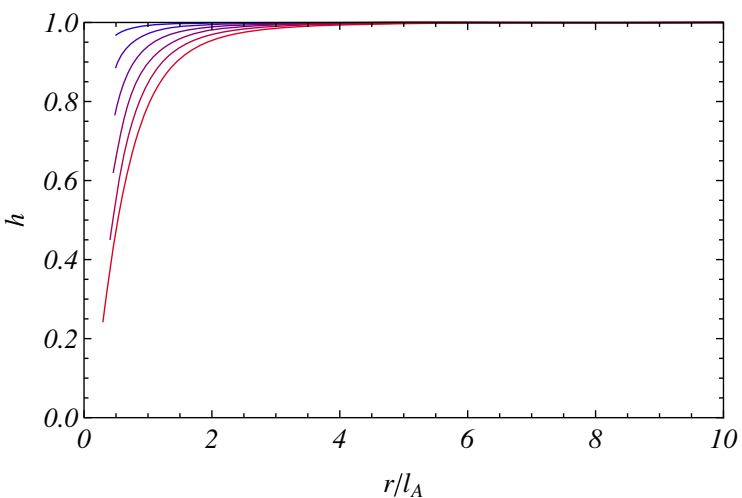

(b)

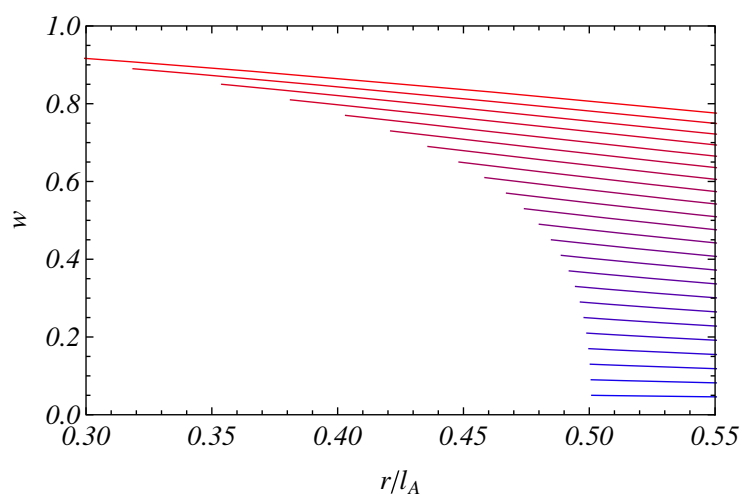

(c)

FIG. 5. (a) The gauge potential $w(\tilde{r})$ and in terms of the radius $\tilde{r}$. We set $\bar{\lambda}=0.1, \tilde{v}=0.1$, and $\tilde{\Lambda}=-1$. We choose $w_{H}=0.01$, $0.16,0.31,046,061,0.76$, and $w_{H}=0.91$ from the bottom curve to the top one. (b) The Higgs field $h(\tilde{r})$ with the same values of $w_{H}$ from the top curve to the bottom one. In the limit of $r_{H} \rightarrow 0$, $w_{H}$ increases while $h_{H}$ decreases. The solution approaches the gravitating monopole. We also show the near horizon behavior of $w(\tilde{r})$ in (c). The curves correspond to the boundary values of $w_{H}=0.01 \sim 0.93$, with an interval of 0.04. $w_{H}$ decreases as the horizon radius gets larger.

We set $\bar{\lambda}=0.1, \tilde{v}=0.1$, and $\tilde{\Lambda}=-1$. In the limit of $r_{H} \rightarrow 0, w_{H}$ increases, while $h_{H}$ decreases. The solution approaches the gravitating monopole. The qualitative behaviors of $w(\tilde{r})$ and $h(\tilde{r})$ are similar to those of the 
SHOICHIRO MIYASHITA and KEI-ICHI MAEDA

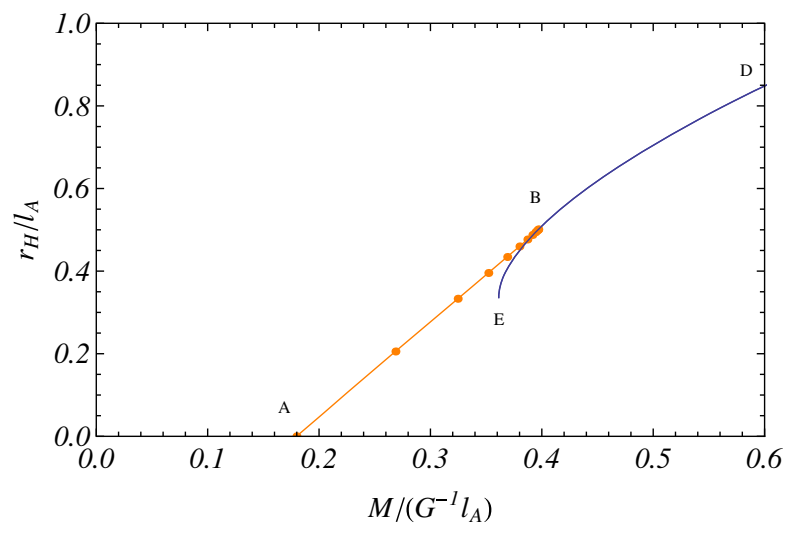

FIG. 6. The $\tilde{M}-\tilde{r}_{H}$ relation for the case of $\tilde{\Lambda}=-1$. The blue curve denotes the RN AdS BH.

monopole $\mathrm{BH}$ without a cosmological constant, which are summarized in Appendix A. As $w_{H}$ decreases, the gauge potential $w(r)$ drops faster to zero and the Higgs field $h(r)$ increases more rapidly to unity. It means that the size of the monopole structure gets smaller because the black hole swallows some portion of the fields. We also show the near horizon behavior of the gauge potential $w(\tilde{r})$ in Fig. 5(c). As $w_{H}$ decreases, the horizon radius $r_{H}$ increases. We find that there exists a maximum radius of the horizon in the limit of $w_{H} \rightarrow 0$. We show the $\tilde{M}-\tilde{r}_{H}$ relations in Fig. 6 . The monopole $\mathrm{BH}$ solution reaches the maximum horizon radius, where the monopole $\mathrm{BH}$ branch connects with the RN AdS BH one.

For the other value of a cosmological constant, we may find different behavior. In Fig. 7, we show the near horizon behavior of $w(\tilde{r})$ for the case of $\tilde{\Lambda}=-0.1$. As $w_{H}$ decreases, $r_{H}$ increases and reaches a maximum at a finite value of $w_{H}=0.447$, then decreases again. In the range of the horizon radius of $0.576 \ell_{A}<r_{H}<0.591 \ell_{A}$, there are

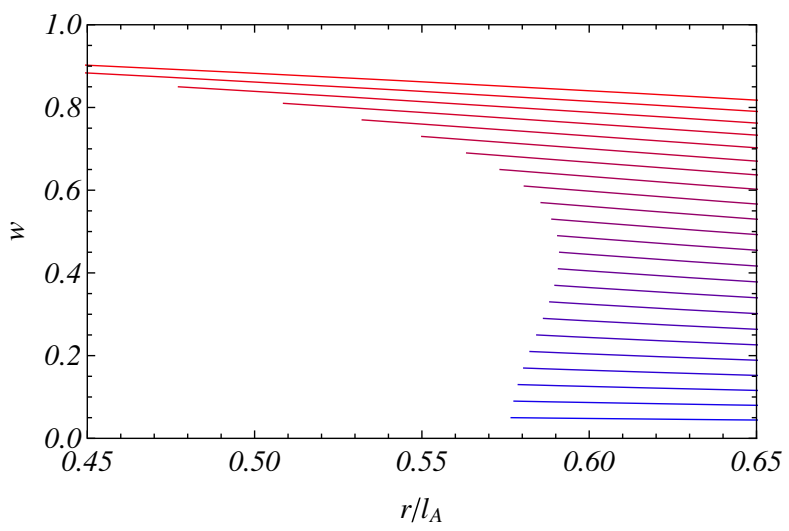

FIG. 7. The near horizon behavior of the gauge potential $w(\tilde{r})$. We set $\bar{\lambda}=0.1$ and $\tilde{v}=0.1$ for $\tilde{\Lambda}=-0.1$. The curves correspond to the boundary values of $w_{H}=0.01-0.93$, with an interval of 0.04. This shows a more complicated relation between the boundary values and the horizon radii.
PHYSICAL REVIEW D 94, 124037 (2016)

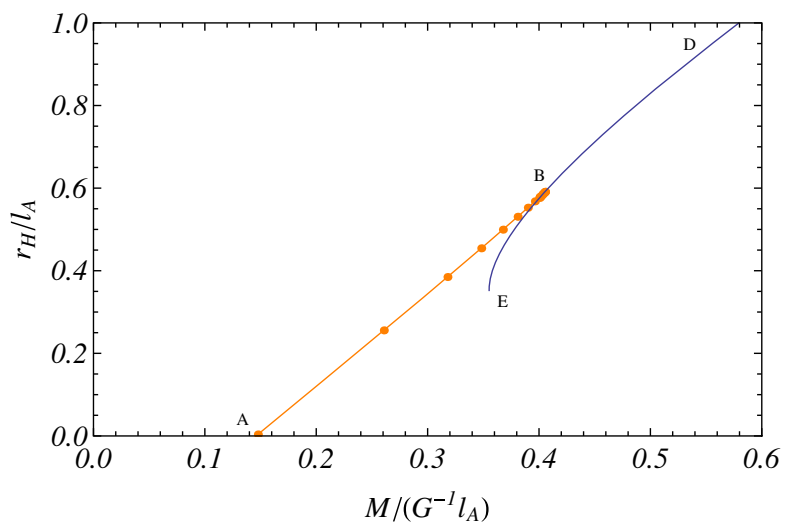

FIG. 8. The $\tilde{M}-\tilde{r}_{H}$ relation for the case of $\tilde{\Lambda}=-0.1$.

two solutions with different values of $w_{H}$. We show the $\tilde{M}-\tilde{r}_{H}$ relations in Fig. 8.

Since the behavior of the monopole $\mathrm{BH}$ branch is more complicated in this case, we provide an enlargement near the junction point in order to display detail. We show the difference between the radii of the monopole $\mathrm{BH}$ and $\mathrm{RN}$ AdS BH in Fig. 9. We find there are three $\mathrm{BH}$ solutions with the same mass in the mass range of $0.4004 \ell_{A}<G M<0.4054 \ell_{A}$, which breaks the $\mathrm{BH}$ uniqueness. We find a cusp structure to which we may apply a catastrophe theory to understand the BH stability, just as in the case without a cosmological constant [10].

From the above two examples, we understand that there exists a critical value of the cosmological constant $\tilde{\Lambda}=$ $\tilde{\Lambda}_{\operatorname{cr}(1)}(\tilde{v})$ which depends on $\tilde{v}$, beyond which the $\tilde{M}-\tilde{r}_{H}$ relation is monotonic; i.e., only one monopole exists for a

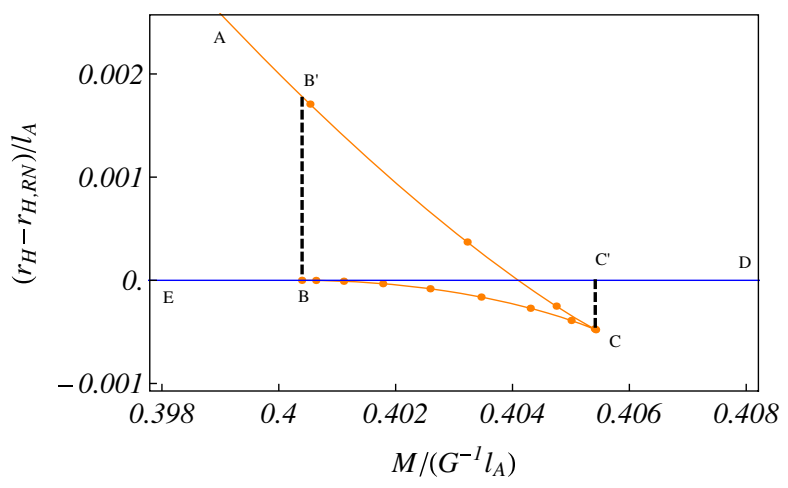

FIG. 9. The enlarged figure of Fig. $8(\tilde{\lambda}=0.1, \tilde{v}=0.1$, and $\tilde{\Lambda}=-0.1)$ near the junction. The vertical axis gives the difference of the horizon radius of the monopole $\mathrm{BH}$ from the $\mathrm{RN}$ AdS $\mathrm{BH}$ one. The blue curve denotes the RN AdS BH. When the stable RN AdS BH with larger mass reduces its mass via Hawking radiation, the evolutionary path goes from $\mathrm{D} \rightarrow \mathrm{B}$, then jumps up to $\mathrm{B}^{\prime} \rightarrow \mathrm{A}$. However, if the stable monopole $\mathrm{BH}$ with smaller mass increases its mass via the mass accretion, the evolutionary path goes from $\mathrm{A} \rightarrow \mathrm{C}$, then jumps from $\mathrm{C}^{\prime} \rightarrow \mathrm{D}$. These are the first order phase transitions between two BHs. 
given mass $M$. In the asymptotically flat case $(\tilde{\Lambda}=0)$, we find similar behavior when we change the value of $\tilde{v}$; i.e., the $\tilde{M}-\tilde{r}_{H}$ relation is monotonic if $\tilde{v}$ is smaller than the critical value. Otherwise, two monopole BH solutions exist and the $\tilde{M}-\tilde{r}_{H}$ relation shows a cusp structure (see Appendix A for details). In the asymptotically AdS case, we have the critical value of $\tilde{\Lambda}_{\operatorname{cr}(1)}(\tilde{v})$ (or the critical curve in the $v$ - $\Lambda$ plane).

We show the $M-r_{H}$ relation for various values of $\tilde{\Lambda}$ in Fig. 10. For smaller values of $|\tilde{\Lambda}|$, we find three $\mathrm{BH}$ solutions near the junction point. When $|\tilde{\Lambda}|$ increases beyond $\left|\tilde{\Lambda}_{\operatorname{cr}(1)}\right|$, the horizon radius of the monopole $\mathrm{BH}$ increases monotonically and then connects with the RN AdS BH branch. As $|\tilde{\Lambda}|$ increases further, the junction point disappears at the second critical value of the cosmological constant $\tilde{\Lambda}_{\text {cr(2) }}$. There exists a gap between the horizon radii

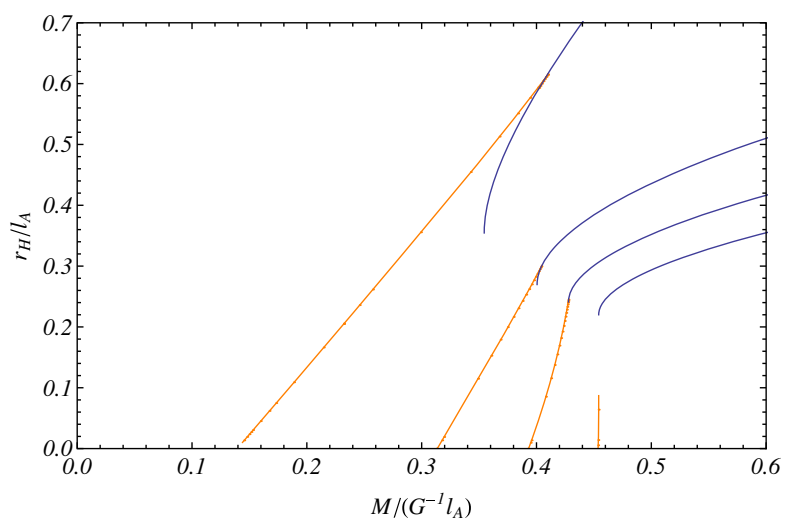

(a)

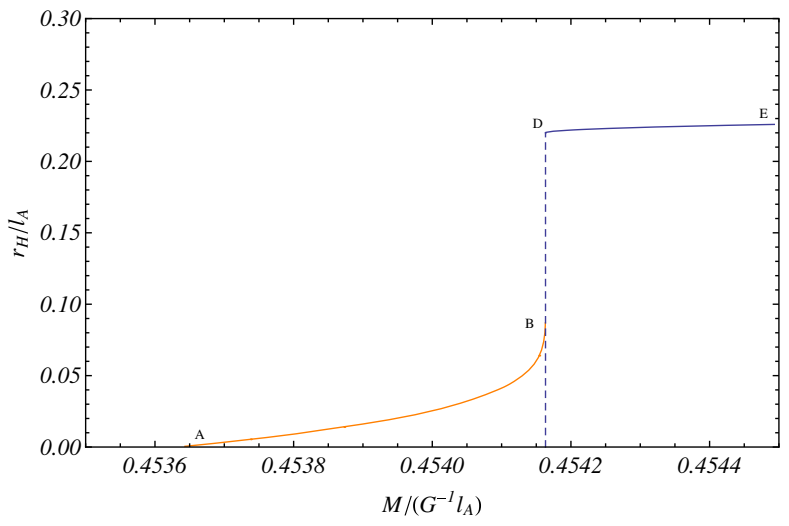

(b)

FIG. 10. (a) $M-r_{H}$ relation in the case where $\bar{\lambda}=0.1$ and $\tilde{v}=0.1$. We choose $\tilde{\Lambda}=0,-10,-20$, and -33 . The blue and orange branches represent the $\mathrm{RN}$ AdS $\mathrm{BH}$ and the AdS monopole BH. We find $\tilde{\Lambda}_{\operatorname{cr}(1)} \approx-0.68, \tilde{\Lambda}_{\operatorname{cr}(2)} \sim-31$, and $\tilde{\Lambda}_{\mathrm{b}} \approx-33.66$. (b) An enlarged figure of the $M-r_{H}$ relations for the case of $\tilde{\Lambda}=-33$. The dashed line represents the mass of an extreme RN AdS BH.

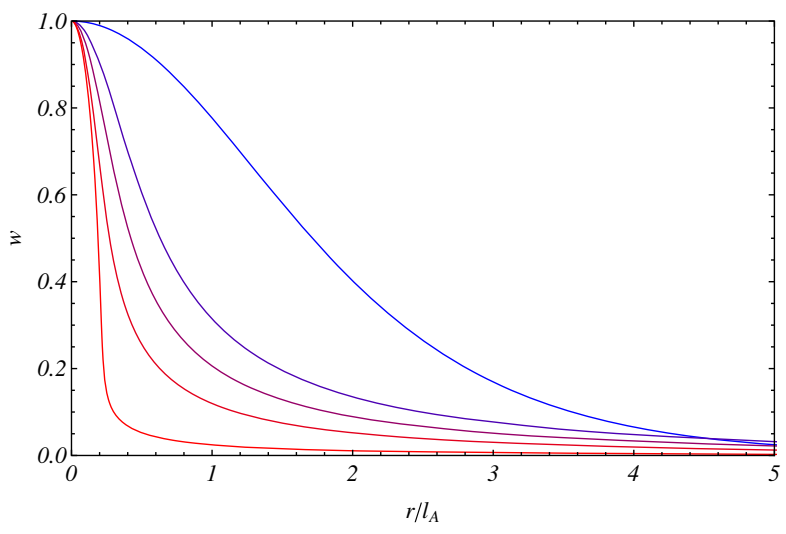

FIG. 11. The behavior of $w(\tilde{r})$ fixing $\tilde{r}_{H}=0.01, \tilde{v}=0.1$ and $\bar{\lambda}=0.1$ but varying $\tilde{\Lambda} . \tilde{\Lambda}=0,-10,-20,-30$ and $\tilde{\Lambda}_{\mathrm{b}} \approx-33.66$ from the top.

of the monopole $\mathrm{BH}$ and of the RN AdS $\mathrm{BH}$ for the cosmological constant of $|\tilde{\Lambda}|>\left|\tilde{\Lambda}_{\mathrm{cr}(2)}\right|$.

Furthermore, if $|\tilde{\Lambda}|$ grows larger, there is a boundary value of the cosmological constant beyond which no monopole exists. Thus, we expect that no monopole $\mathrm{BH}$ exists either. Figure 11 depicts $w(\tilde{r})$ for different values of $\tilde{\Lambda}$ with the same horizon radius: $\tilde{r}_{H}, \bar{\lambda}$, and $\tilde{v}$. We find that $w(\tilde{r})$ drops faster to zero as $|\tilde{\Lambda}|$ increases. This shows that the radius of the monopole structure grows smaller with the existence of a negative cosmological constant, which we have found in the gravitating monopole, too. Figure 12 shows the behavior of $f(\tilde{r})$ in the case of Fig. 11. As $|\tilde{\Lambda}|$ grows larger, the minimum decreases and eventually reaches zero, resulting in an extreme $\mathrm{RN} \mathrm{BH}$. Then the monopole $\mathrm{BH}$ solution and the gravitating monopole solution disappear beyond the boundary value $\tilde{\Lambda}_{\mathrm{b}}$.

We conclude that the effect of $\tilde{\Lambda}$ is similar to that of $\tilde{v}$, as claimed in the work on the AdS monopole [27,28], and there exists a boundary value $\tilde{\Lambda}_{\mathrm{b}}$ beyond which there is no

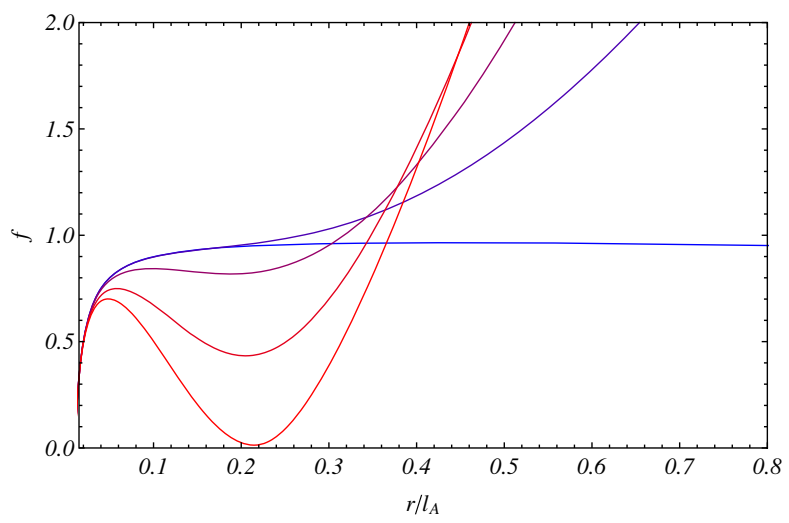

FIG. 12. The behavior of $f(\tilde{r})$ fixing $\tilde{r}_{H}=0.01, \tilde{v}=0.1$, and $\bar{\lambda}=0.1$, but varying $\tilde{\Lambda} . \tilde{\Lambda}=0,-10,-20,-30$ and $\tilde{\Lambda}_{\mathrm{b}} \approx-33.66$ from the top. 
monopole $\mathrm{BH}$ solution, as well as two critical values, $\tilde{\Lambda}_{\operatorname{cr}(1)}$ and $\tilde{\Lambda}_{\mathrm{cr}(2)}$. This feature is important in the BH thermodynamics and the phase transition, which we will discuss in the next section.

\section{BLACK HOLE THERMODYNAMICS AND PHASE TRANSITION}

As is well known, the dynamics of a black hole obeys thermodynamical laws [19]. Its dynamical stability may be closely related to thermodynamical stability, and it may be understood by a catastrophe theory for hairy black holes [10]. Hence, we analyze the thermodynamical variables and discuss the thermodynamical stability and the possible phase transitions.

First, we define the thermodynamical variables. BH entropy is given by the Bekenstein-Hawking area-entropy relation:

$$
S=\frac{\mathcal{A}_{H}}{4 G}=\frac{\pi r_{H}^{2}}{G},
$$

where $\mathcal{A}_{H}=4 \pi r_{H}^{2}$ is the area of the event horizon. For the convenience of our numerical calculation, we will use the following normalized dimensionless entropy:

$$
\tilde{S}=e^{2} S=\frac{\pi \tilde{r}_{H}^{2}}{\tilde{v}^{2}}
$$

The Hawking temperature is given by

$$
T=\frac{f^{\prime}\left(r_{H}\right)}{4 \pi} e^{-\delta\left(r_{H}\right)},
$$

and the dimensionless temperature $\tilde{T}$ is defined by

$$
\tilde{T} \equiv \frac{\sqrt{G}}{e} T=\left.\frac{\tilde{v}}{4 \pi} \frac{d f(\tilde{r})}{d \tilde{r}}\right|_{\tilde{r}=\tilde{r}_{H}} e^{-\delta\left(\tilde{r}_{H}\right)}
$$

In the following subsections, we shall discuss the thermodynamical properties of an isolated system and those in a thermal heat bath separately.

\section{A. Isolated system}

First, we consider an isolated system. The BH entropy is an indicator for the thermal instability. According to the second law of thermodynamics, the entropy in an isolated system never decreases and a maximum entropy state is realized. In the $\mathrm{BH}$ thermodynamics, when there are several $\mathrm{BH}$ solutions with the same mass, which corresponds to an internal energy in thermodynamics, the $\mathrm{BH}$ with maximum entropy is thermodynamically stable. However, this does not mean that the other $\mathrm{BH}$ is always unstable. Although it is not globally stable, it can be locally stable. This local thermodynamical stability may correspond to the dynamical stability of BHs. Although there is no proof, no counterexample is known.

Since the BH entropy is proportional to the horizon area $\left(\tilde{\mathcal{A}}_{H}=4 \pi \tilde{r}_{H}^{2}\right)$, the $\tilde{M}-\tilde{r}_{H}$ relation is sufficient to analyze the thermodynamical stability. A catastrophe theory may also provide a good tool to judge the stability [10].

Classifying the value of the cosmological constant into the following three cases, we shall discuss the behavior of the horizon radius and the $\mathrm{BH}$ phase transition separately.

$\tilde{\Lambda}_{\operatorname{cr}(1)}(\tilde{v})<\tilde{\Lambda}(\leq 0)$. - There are two monopole BH solutions as well as RN AdS BH. We find a cusp structure in the $\tilde{M}-\tilde{r}_{H}$ relation.

In Fig. 9, we show the $\tilde{M}-\tilde{r}_{H}$ relation for the case where $\bar{\lambda}=0.1, \tilde{v}=0.1$, and $\tilde{\Lambda}=-0.1$ (Note that $\tilde{\Lambda}_{\operatorname{cr}(1)} \approx-0.68$.) The RN AdS BH and the monopole $\mathrm{BH}$ are stable on the intervals of $\mathrm{BD}$ and $\mathrm{AC}$, respectively. When the stable RN AdS BH with larger mass reduces its mass via the Hawking radiation, the evolutionary path goes from $\mathrm{D} \rightarrow \mathrm{B}$, then jumps from $\mathrm{B}^{\prime} \rightarrow \mathrm{A}$. However, if the stable monopole $\mathrm{BH}$ with smaller mass increases its mass via mass accretion, the evolutionary path goes from $\mathrm{A} \rightarrow \mathrm{C}$, then jumps from $\mathrm{C}^{\prime} \rightarrow \mathrm{D}$. These are the first order phase transitions between two BHs.

$\tilde{\Lambda}_{\operatorname{cr}(2)}(\tilde{v})<\tilde{\Lambda}<\tilde{\Lambda}_{\operatorname{cr}(1)}(\tilde{v})$. - There is one monopole $\mathrm{BH}$ solution, as well as RN AdS BH. The monopole BH branch connects with the RN AdS BH branch. This is no cusp structure in the $\tilde{M}-\tilde{r}_{H}$ relation, as shown in Fig. 6, for which we have chosen $\bar{\lambda}=0.1, \tilde{v}=0.1$, and $\tilde{\Lambda}=-1$ (Note that $\tilde{\Lambda}_{\text {cr(2) }} \sim-31$.) The RN AdS BH is unstable in the intervals of BE. As a result, the evolutionary path when the mass changes is either $\mathrm{A} \rightarrow \mathrm{B} \rightarrow \mathrm{D}$ or the reverse. This is the second order phase transition between two BHs.

$\tilde{\Lambda}_{\mathrm{b}}(\tilde{v})<\tilde{\Lambda}<\tilde{\Lambda}_{\operatorname{cr}(2)}(\tilde{v})$. - There is one monopole $\mathrm{BH}$ solution, as well as RN AdS BH, but those two branches are disconnected, as shown in Fig. 10(b), for which we have chosen $\bar{\lambda}=0.1, \tilde{v}=0.1$, and $\tilde{\Lambda}=-33$. (Note that $\tilde{\Lambda}_{\mathrm{b}} \approx-33.66$.) A radius gap at the extreme states is present between the two branches. The evolutionary path when the mass increases goes from $\mathrm{A} \rightarrow \mathrm{B}$, then jumps from $\mathrm{D} \rightarrow \mathrm{E}$. This may be the nonreversible first order phase transition.

$\tilde{\Lambda}<\tilde{\Lambda}_{\mathrm{b}}(\tilde{v})$.- There is no monopole BH solution. Only a stable RN AdS BH exists. No phase transition occurs.

In order to see the effect of $\tilde{\Lambda}$ on the phase transition strength, we analyze the magnitude of entropy discontinuity. Comparing the $\tilde{M}-\tilde{r}_{H}$ relations in Fig. $9(\tilde{\Lambda}=-0.1)$ with Fig. $24(\tilde{\Lambda}=0)$, we find that the cusp grows smaller as $|\tilde{\Lambda}|$ gets bigger.

Figure 13 confirms this fact. The vertical axis shows the discontinuity of $\mathrm{BH}$ entropies between the monopole $\mathrm{BH}$ at the cusp point $\mathrm{C}$ and $\mathrm{RN}$ AdS BH. It shows how the cusp structure becomes small and eventually vanishes at the 


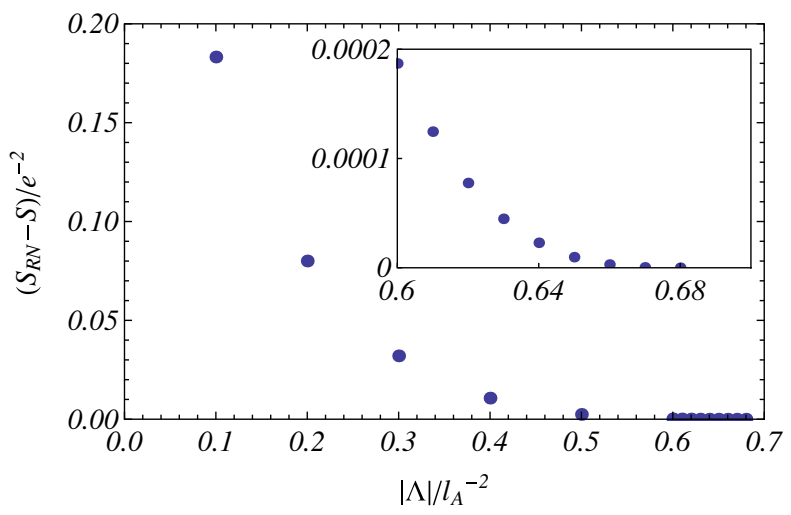

FIG. 13. $\tilde{\Lambda}$ dependence of the discontinuity of the BH entropy between the monopole $\mathrm{BH}$ and $\mathrm{RN}$ (AdS) $\mathrm{BH}$ at the cusp point. We set $\bar{\lambda}=0.1$ and $\tilde{v}=0.1$, for which we find $\tilde{\Lambda}_{\operatorname{cr}(1)} \approx-0.68$. (Inset) Enlargement of the main figure near the transition point.

critical point $\tilde{\Lambda}_{\operatorname{cr}(1)} \approx-0.68$. We also show how the $\mathrm{BH}$ temperatures depend on the cosmological constant in Fig. 14.

At the critical value of the cosmological constant $\tilde{\Lambda}_{\operatorname{cr}(1)}$, these two curves cross. This is consistent with our claim that the phase transition changes from first order to second order at the critical value $\tilde{\Lambda}_{\operatorname{cr}(1)}$.

In addition, at the second critical value of the cosmological constant $\tilde{\Lambda}_{\text {cr(2) }}$, another change of the types of phase transition is expected. Beyond $\tilde{\Lambda}_{\operatorname{cr}(2)}$, the monopole $\mathrm{BH}$ evolves into the extreme RN AdS BH, as shown in Fig. 15. When the minimum of $f(\tilde{r})$ vanishes as $\tilde{r}_{H}$ increases, the event horizon of the extreme RN AdS BH appears at a bigger horizon radius $\left(\tilde{r}_{H} \approx 0.220\right)$ than that of the monopole BH $\left(\tilde{r}_{H} \approx 0.091\right)$. Then the first order phase transition occurs with the discontinuous changes of the $\mathrm{BH}$ entropy and the $\mathrm{BH}$ temperature. Note that the $\mathrm{BH}$ temperatures of the monopole $\mathrm{BH}$ at the transition point and of the extreme

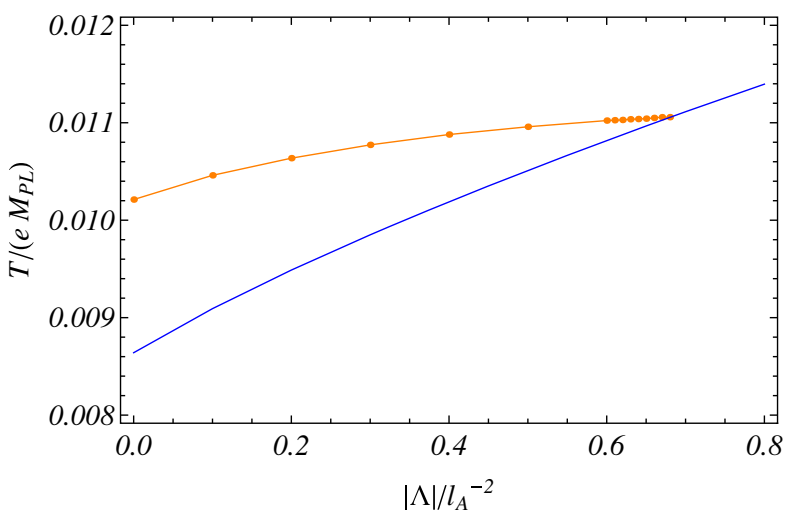

FIG. 14. The BH temperatures with respect to $\tilde{\Lambda}$. We set $\bar{\lambda}=$ 0.1 and $\tilde{v}=0.1$. The orange branch and the blue one denote the monopole $\mathrm{BH}$ temperature and the $\mathrm{RN}$ AdS $\mathrm{BH}$ one, respectively.

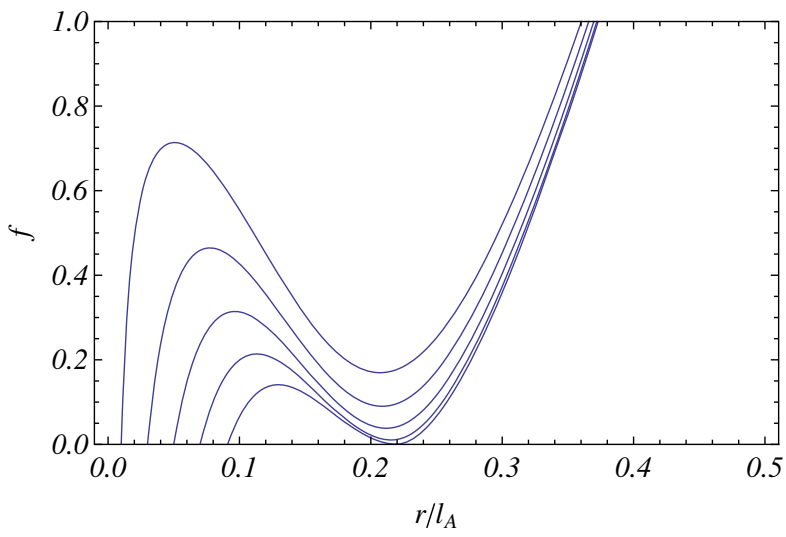

FIG. 15. The behavior of $f(\tilde{r})$ fixing $\tilde{\Lambda}=-33, \tilde{v}=0.1$, and $\bar{\lambda}=0.1$ but varying $\tilde{r}_{H} \cdot \tilde{r}_{H}=0.01,0.03,0.05,0.07$, and 0.091 from the top.

RN AdS BH are $\tilde{T} \sim O\left(10^{-5}\right) \neq 0$ and $\tilde{T}=0$, respectively. The inverse process from the RN AdS BH to the monopole $\mathrm{BH}$ may not occur, because the BH entropy must decrease at the transition point.

The above two critical values, $\tilde{\Lambda}_{\operatorname{cr}(1)}$ and $\tilde{\Lambda}_{\mathrm{cr}(2)}$, as well as the boundary value, $\tilde{\Lambda}_{\mathrm{b}}$, depend on both $\tilde{v}$ and $\bar{\lambda}$. Fixing $\bar{\lambda}=0.1$, we show them in Fig. 16.

When we increase the coupling constant $\bar{\lambda}$, region I will shrink and vanish-for example, in the case of $\bar{\lambda}=1$.

\section{B. Black holes in a heat bath}

Next, we consider thermodynamical stability of BHs in a heat bath. We deal with two thermodynamical variables. One is the heat capacity, while the other is the Helmholtz free energy. The heat capacity is defined by

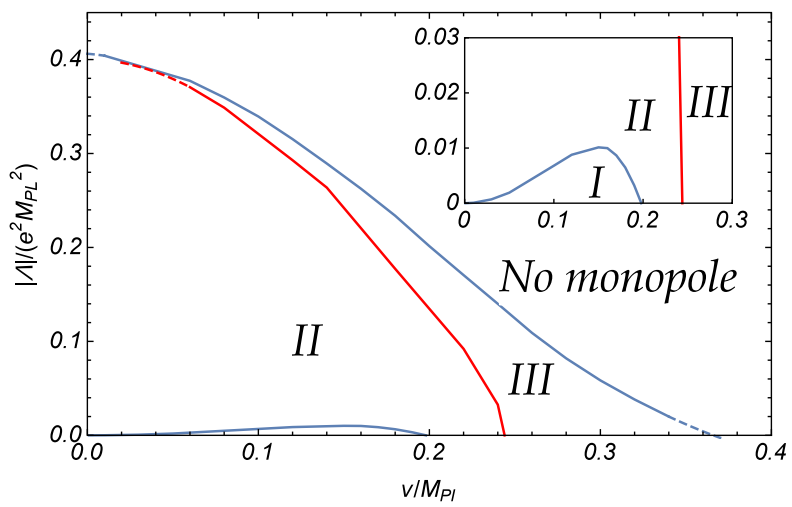

FIG. 16. The $v$ dependence of $\Lambda_{\operatorname{cr}(1)}, \Lambda_{\operatorname{cr}(2)}$, and $\Lambda_{\mathrm{b}}$ for the case of $\bar{\lambda}=0.1$. The curves correspond to the cases of $\Lambda_{\operatorname{cr}(1)}, \Lambda_{\operatorname{cr}(2)}$, and $\Lambda_{\mathrm{b}}$, respectively, from the bottom. Region I shows where the first order phase transition occurs, while region II denotes where the second order phase transition is expected. In region III, we find the first order phase transition from the monopole $\mathrm{BH}$ to the extreme RN AdS BH. Beyond $\Lambda_{\mathrm{b}}$, no monopole $\mathrm{BH}$ exists. 


$$
C=T\left(\frac{\partial S}{\partial T}\right) .
$$

If the heat capacity is negative, a system in a heat bath is thermodynamically unstable, while such a state is locally stable when the system has a positive heat capacity. For the RN AdS BH, its behavior is well studied [22]. As shown in Figs. 17 and 18, a finite intermediate range of the entropy exists where the heat capacity becomes negative. Hence, the RN AdS BH becomes unstable in such a range. The unstable BH may change its entropy (the horizon radius) via the evaporation or accretion and evolve into a stable one. As for the monopole BH branch, the solution appears at some point of the RN AdS BH branch. It may change the evolutional path.

In the asymptotically flat case, the monopole $\mathrm{BH}$ in a heat bath is locally unstable for almost all of the parameters. It is because the entropy of the monopole $\mathrm{BH}$ usually decreases monotonically as the $\mathrm{BH}$ temperature increases, giving the negative heat capacity. However, when $\bar{\lambda}$ is small enough, there is a thermodynamically stable monopole $\mathrm{BH}$ in a narrow range of the temperature [11]. The parameter region where the stable monopole BHs exist coincides with one where the cusp structure appears in the $\tilde{M}-\tilde{r}_{H}$ relation. This is because when there are two monopole BHs with the same horizon radius $r_{H}$ (equivalently, the same entropy), the $\mathrm{BH}$ entropy must increase in some parameter region. We find the same feature for the AdS monopole BHs when $|\tilde{\Lambda}|$, as well as $\bar{\lambda}$ and $\tilde{v}$, is small.

We show some examples in Fig. 17. We choose the parameters $\bar{\lambda}=0.1, \tilde{v}=0.1$, and $\tilde{\Lambda}=-0.1$. The monopole $\mathrm{BH}$ branch appears near the marginally stable point of RN AdS BHs. The heat capacity is first positive near the junction point, but it quickly becomes negative when the temperature increases. Hence, there exists a stable monopole $\mathrm{BH}$ solution for a narrow range of temperature. This happens when $\tilde{\Lambda}>\tilde{\Lambda}_{\text {cr(1) }}$.

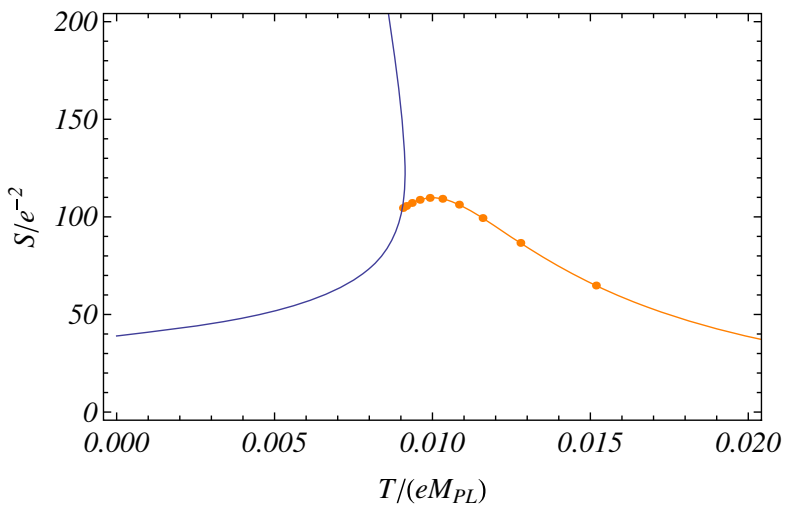

FIG. 17. $\tilde{T}-\tilde{S}$ relation. $(\bar{\lambda}=0.1, \tilde{v}=0.1$, and $\tilde{\Lambda}=-0.1$.) The blue line represents $\mathrm{RN} \mathrm{BH}$ and the orange line represents the monopole $\mathrm{BH}$.

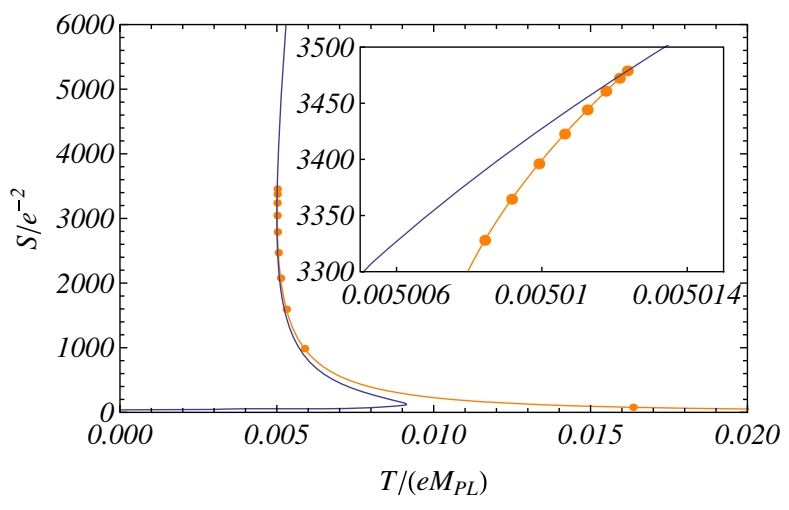

FIG. 18. $\tilde{T}-\tilde{S}$ relation. $(\bar{\lambda}=0.1, \tilde{v}=0.001$, and $\tilde{\Lambda}=-1000$. $)$ The blue line represents $\mathrm{RN} \mathrm{BH}$ and the orange line represents the monopole $\mathrm{BH}$.

When $\tilde{\Lambda}_{\operatorname{cr}(1)}>\tilde{\Lambda}>\tilde{\Lambda}_{\mathrm{b}}$, the entropy of monopole BH decreases monotonically in terms of temperature. As a result, there is no thermodynamically stable monopole $\mathrm{BH}$.

In addition, we find new behaviors of thermodynamical stability when $\tilde{v}$ is very small but $|\tilde{\Lambda}|$ is large enough. As shown in Fig. 18, the heat capacity changes its sign through infinity. A similar stability behavior is found in the case of an Einstein-SU(2)-Yang-Mills system with a negative cosmological constant [26]. This may be explained by the fact that the monopole $\mathrm{BH}$ solution near the $\mathrm{RN} \mathrm{AdS} \mathrm{BH}$ is only slightly affected by the Higgs field.

Next, we discuss the Helmholtz free energy $F$, which is another thermodynamical indicator. We may judge a global stability by this indicator, as we will see. Note that the heat capacity is the indicator for judging a local stability of a system in a heat bath.

For convenience, we introduce a dimensionless free energy defined by

$$
\tilde{F} \equiv e \sqrt{G} F
$$

In the Einstein-Maxwell theory with a negative cosmological constant, it is known that, if $|\Lambda|$ is small enough, we find the first order phase transition between the large RN AdS BH and the small RN AdS BH in a thermal bath system [22]. The "swallow tail" structure appears in the $F-T$ plane in this phase transition. In an $\mathrm{SO}(3) \mathrm{EYMH}$ system with a negative cosmological constant, there are three thermal states: a RN AdS BH in a thermal bath, a monopole $\mathrm{BH}$ in a thermal bath, and a thermal monopole. A thermal monopole is a gravitating monopole in a heat bath with an arbitrary temperature, which is as shown in Appendix B.

We show these Helmholtz free energies in Figs. 19 and 20 for $\tilde{\Lambda}=-1$ and $\tilde{\Lambda}=-0.1$, respectively. For the large cosmological constant $(\tilde{\Lambda}=-1)$, as shown in Fig. 19, no swallow tail appears, while, for the small cosmological 


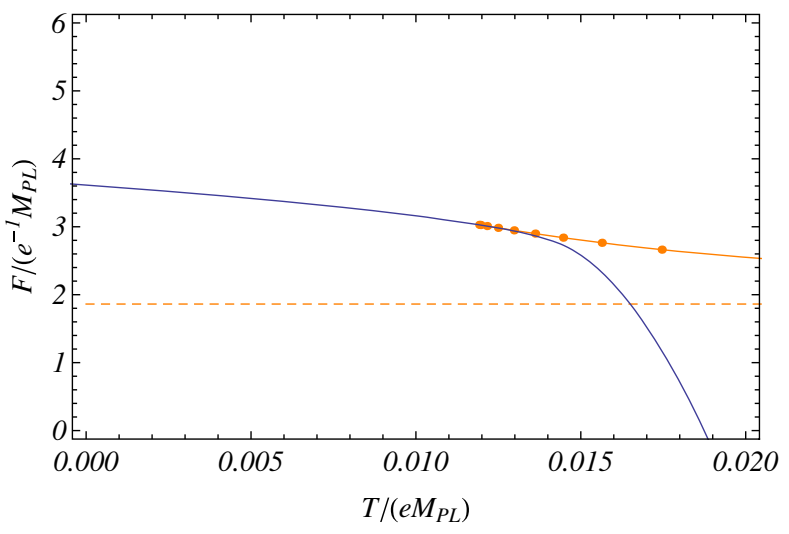

FIG. 19. $\tilde{T}-\tilde{F}$ relation. $(\bar{\lambda}=0.1, \tilde{v}=0.1$, and $\tilde{\Lambda}=-1$. $)$ The blue line represents the $\mathrm{RN}$ AdS $\mathrm{BH}$ and the orange line represents the monopole $\mathrm{BH}$. The orange dashed line represents a monopole.

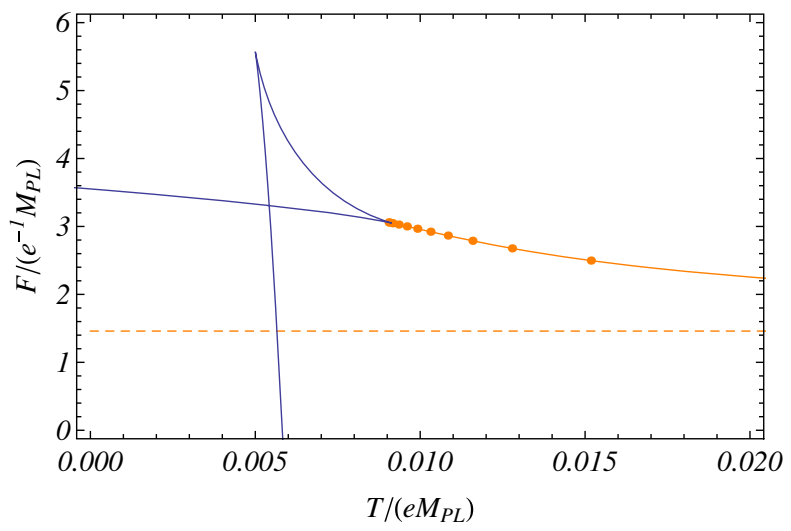

FIG. 20. $\tilde{T}$ - $\tilde{F}$ relation. $(\bar{\lambda}=0.1, \tilde{v}=0.1$, and $\tilde{\Lambda}=-0.1$.) The blue line represents RN BH and the orange solid line represents monopole $\mathrm{BH}$. The orange dashed line represents a monopole.

constant $(\tilde{\Lambda}=-0.1)$, we find a swallow tail structure in Fig. 20. As we have discussed for the heat capacity, there is a thermodynamically stable monopole BH. However, it is just locally stable. As seen from Figs. 19 and 20, the monopole is favored in the low temperature region, while the RN AdS BH becomes mostly stable in the high temperature region. The monopole $\mathrm{BH}$ is never favored in a thermal bath. As a result, the Hawking-Page phase transition may occur only between the monopole and the RN AdS BH.

\section{CONCLUDING REMARKS}

We have analyzed the properties of magnetically charged, asymptotically AdS spacetime in the EYMH system and its thermodynamical properties. Studying the thermodynamical stability, we have discussed the types of phase transition for an isolated system and for the system in a heat bath.
The type of phase transition depends on $\tilde{\Lambda}$ as well as $\tilde{v}$ and $\bar{\lambda}$ of the Higgs field. In the case of an isolated system, keeping $\bar{\lambda}$ small $[\bar{\lambda} \lesssim O(1)]$, we find two critical values and one boundary value of the cosmological constant, $\tilde{\Lambda}_{\operatorname{cr}(1)}$, $\tilde{\Lambda}_{\mathrm{cr}(2)}$, and $\tilde{\Lambda}_{b}$, which depend on $\tilde{v}$. The phase diagram on the $\tilde{v}$ - $\tilde{\Lambda}$ parameter plane is given by Fig. 16. For the large value of $\bar{\lambda} \gg O(1)$, although the extreme monopole $\mathrm{BH}$ was found in the case of $\Lambda=0$ (see the schematic diagram in Fig. 27), we could not confirm the existence of such a solution because of numerical difficulty.

In the case of a $\mathrm{BH}$ system in a heat bath, we find the Hawking-Page transition between the RN AdS BH and the gravitating monopole. The monopole $\mathrm{BH}$ does not play anything in this transition, although it is locally stable.

This Hawking-Page transition describes a transition between a zero-entropy soliton and a finite-entropy $\mathrm{BH}$ spacetime. Such a phase transition was first discussed in the SU(2)-EYM system [25]. They showed the possibility of a phase transition between a Bjoraker-Hosotani soliton and a RN AdS BH. The AdS colored BH, where a hairy BH also exists in the system, does not play anything in the transition when the magnetic charge is unity. Our result of the Hawking-Page phase transition between the AdS monopole and the RN AdS BH is similar to that in the SU(2)-EYM system. The interesting point is that a non-Abelian gauge field leads to the appearance of a zero-entropy soliton state. In the SU(2)-EYM system, since the magnetic charge changes continuously, we can discuss the transition in the large phase diagram. The soliton phase smoothly connects to the thermal AdS solution when the charge decreases to 0. While in our EYMH system, the magnetic charge is quantized. The soliton phase and the AdS space are disconnected. As a result, our phase diagram is restricted. If we extend the present solution to the dyon, however, we may discuss a larger variety of transitions, as with the EYM case.

Since there are many types of phase transitions in the EYMH system, it would be interesting to apply this model to the AdS/CFT correspondence. Since our solution is constructed in four spacetime dimensions, we may discuss 3D quantum field theory (QFT). The QFT in 3D flat spacetime corresponding to the 4D EYMH system was already investigated in $[30,32]$. The authors solved the Julia-Zee dyon BH solution with asymptotically AdS spacetime and investigated its properties mainly in the planar limit with and without a backreaction. They showed that the nontrivial dyon $\mathrm{BH}$ solution is favored at low temperatures, while the $\mathrm{RN}$ dyon $\mathrm{BH}$ solution is favored at high temperatures, fixing the chemical potential and the magnetic charge on the boundary, which corresponds to a grand canonical ensemble. ${ }^{3}$ This phenomenon corresponds

\footnotetext{
${ }^{3}$ Strictly speaking, it is not a "dyon" since the magnetic flux on the boundary vanishes in the planar limit.
} 
to a phase transition between a condensed phase and a normal state of a certain QFT living in $\mathbb{R}^{2} \times \mathbb{R}_{t}^{1}$. Consideration of the holographic dual of our nontrival $\mathrm{BH}$ solution would be interesting because of its variety of phase transitions. This work is in progress.

To explore 4D conformal field theory, we have to extend our work to higher dimensions. The stable (AdS) gravitating monopole in 4D spacetime is just an extension of a 't Hooft-Polyakov monopole which is topologically stable in 4D flat space. Then we must first find a 5D topologically stable "monopole." However, the known higher dimensional generalization of such a monopole is obtained only for even-spacetime dimensions [38]. As a result, a 5D extension of our study is not straightforward, even if it is possible in principle.

\section{ACKNOWLEDGMENTS}

S. M. would like to thank Y. Hoshino and T. Kitamura for the useful discussions. This work was supported, in part, by grants-in-aid from the Scientific Research Fund of the Japan Society for the Promotion of Science (Grants No. 25400276 and No. 16K05362).

\section{APPENDIX A: ASYMPTOTICALLY FLAT MONOPOLE BLACK HOLE}

In this appendix, we consider a case without a cosmological constant $(\Lambda=0)$ and summarize the properties of a monopole black hole in asymptotically flat spacetime. They were discussed in pioneering works [7-17], finding the dependence of the parameters in the theory, which we show below.

\section{Monopole black hole solution}

Figure 21 shows the behavior of the gauge potential $w(\tilde{r})$ near the horizon. We choose the parameters as $\tilde{v}=0.1$, $\bar{\lambda}=1 . w(\tilde{r})$ decreases monotonically and then vanishes at

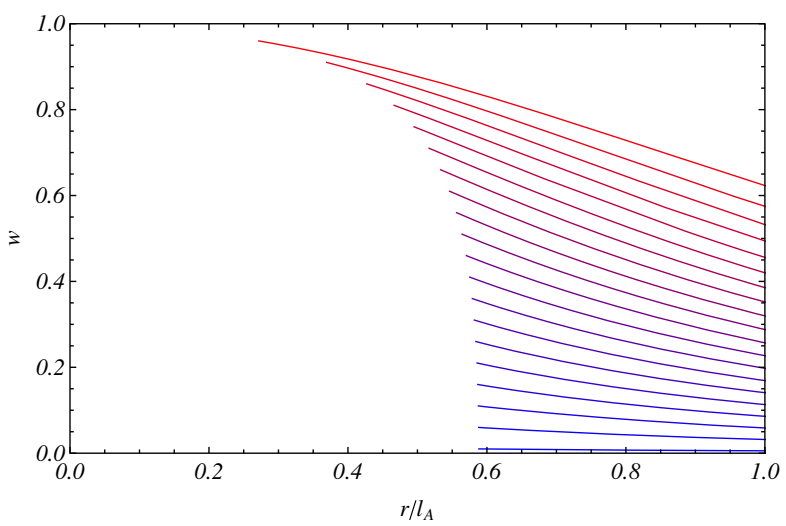

FIG. 21. The near horizon behavior of $w(\tilde{r})$ for $\tilde{v}=0.1$ and $\bar{\lambda}=1$. Each line denotes the solution with $w_{H}=0.01-0.96$ at the interval of 0.05 from the bottom curve.

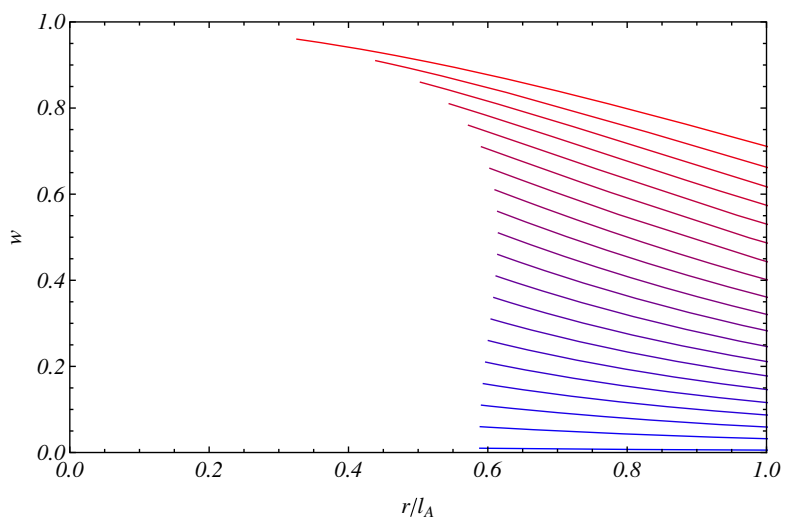

FIG. 22. The near horizon behavior of $w(\tilde{r})$ in the case of $\bar{\lambda}=0.1$ and $\tilde{v}=0.1$. Each line denotes the solution with $w_{H}=0.01-0.96$ at the interval of 0.05 from the bottom curve.

infinity. As shown in Fig. 21, the horizon radius $\tilde{r}_{H}$ grows larger as $w_{H}$ gets smaller.

However, if $\bar{\lambda}$ is small enough, we find more complicated behavior. As shown in Fig. 22, when $\bar{\lambda}=0.1$, there are two monopole $\mathrm{BH}$ solutions with the same horizon radius but different values of $w_{H}$.

We show the $\tilde{M}-\tilde{r}_{H}$ relation in Fig. 23, where we plot two cases of $\bar{\lambda}=0.1$ and 1. A monopole BH branch and a RN $\mathrm{BH}$ one are both present. These two branches connect at one junction point. The two figures look similar, but there exists a cusp structure near the junction point only for the case of $\bar{\lambda}=0.1$, which enlarged figure near the junction point is shown in Fig. 24. There are two monopole BH solutions near the junction point. The vertical axis denotes the difference between the horizon radii of the $\mathrm{RN} \mathrm{BH}$ and the monopole $\mathrm{BH}$.

We should also address the existence of the nontrivial solution. Figure 25 shows the behavior of $f(\tilde{r})$ for a given horizon radius $\tilde{r}_{H}$. As $\tilde{v}$ becomes larger, the minimum value of $f(\tilde{r})$ decreases and eventually vanishes. For a given value of $\bar{\lambda}$, there exists a boundary value $\tilde{v}_{\mathrm{b}}(\bar{\lambda})$, beyond

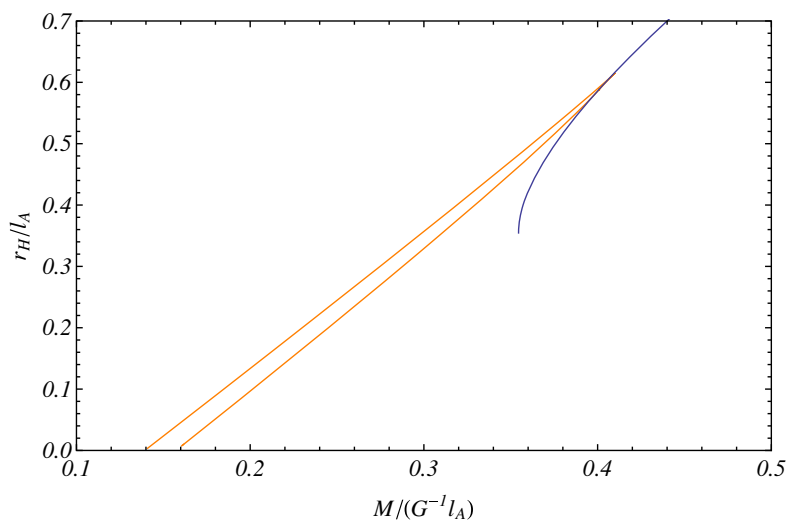

FIG. 23. The $\tilde{M}-\tilde{r}_{H}$ relations for the cases of $\bar{\lambda}=1$ (the lower orange curve) and $\bar{\lambda}=0.1$ (the upper orange curve). We set $\tilde{v}=0.1$. The blue curve denotes the RN BH branch. 


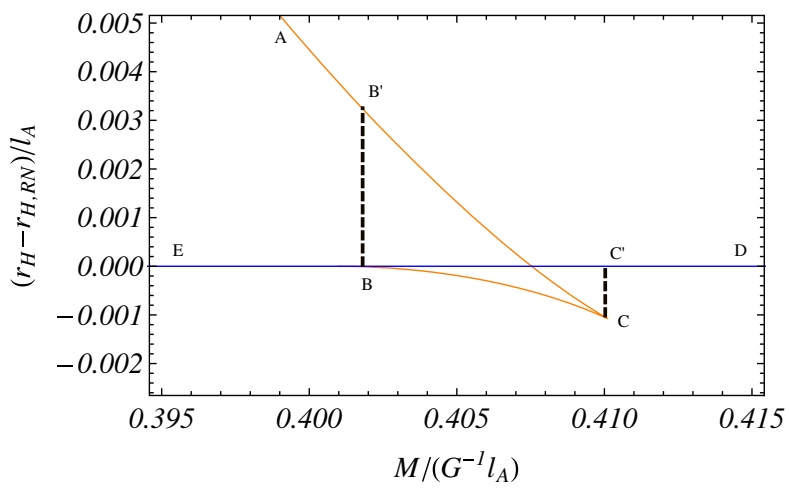

FIG. 24. The enlarged figure of Fig. 23 near the junction point for the cases of $\bar{\lambda}=0.1$ and $\tilde{v}=0.1$. The vertical axis denotes the difference of the horizon radii of the monopole $\mathrm{BH}$ and the $\mathrm{RN}$ $\mathrm{BH}$. There are two monopole $\mathrm{BH}$ solutions near the junction point $\mathrm{B}$. We find a cusp structure. When the large mass $\mathrm{RN} \mathrm{BH}$ reduces its mass via the Hawking radiation, the evolution path is $D \rightarrow B \rightarrow B^{\prime} \rightarrow A$. If the small mass monopole $\mathrm{BH}$ increases its mass via matter accretion, the evolution path is $A \rightarrow C \rightarrow$ $C^{\prime} \rightarrow D$. We expect a first order phase transition in both cases.

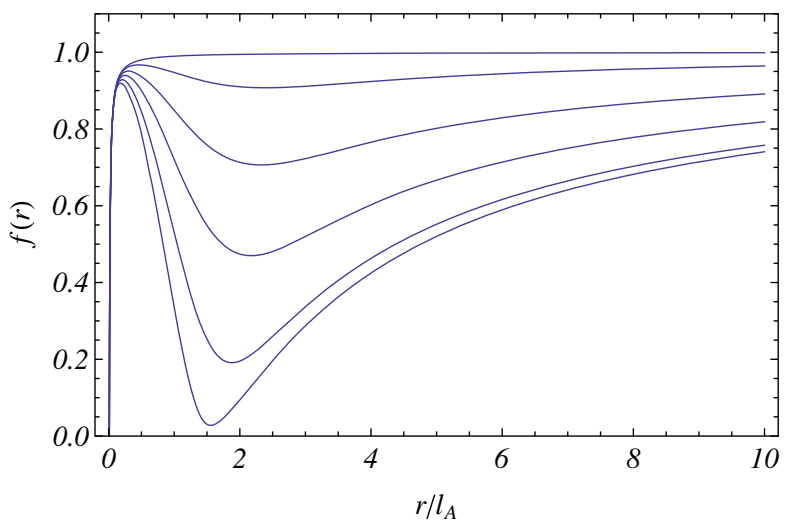

FIG. 25. The behaviors of $f(r)=(1-2 \tilde{m}(\tilde{r}) / \tilde{r})$ with different values of $\tilde{v}$, i.e., $\tilde{v}=0.01,0.12,0.22,0.3,0.37$, and $0.3932 \approx \tilde{v}_{\mathrm{b}}$ from the top. We set $\bar{\lambda}=0, \tilde{r}_{H}=0.01$.

which a gravitating monopole solution ceases to exist. When $f(\tilde{r})$ vanishes, the zero point becomes a horizon of the extreme RN BH. We have only the RN BH solution beyond the critical value $\tilde{v}_{\mathrm{b}}(\bar{\lambda})$.

\section{Thermodynamics}

Next, we discuss thermodynamical properties of a monopole $\mathrm{BH}$ as well as a trivial $\mathrm{RN} \mathrm{BH}$. We consider two cases: an isolated $\mathrm{BH}$ system and a $\mathrm{BH}$ in a thermal bath.

\section{a. Isolated system}

For large $\bar{\lambda}$, there are only two branches: the RN BH branch and the monopole $\mathrm{BH}$ branch (see Fig. 23). In an isolated system, the monopole $\mathrm{BH}$ branch is thermodynamically favored because its entropy is larger than that of the RN BH. Since there is no monopole BH above the junction point, the $\mathrm{RN} \mathrm{BH}$ is unique.

In the present case, thermodynamical stability is equivalent to dynamical instability. It was shown that the monopole $\mathrm{BH}$ solution is always stable against linear perturbations, while the RN BH solution becomes unstable below the junction point, although it is stable above the junction point $[9,12]$. We may understand this fact intuitively because, for such a $\mathrm{RN} \mathrm{BH}$, we have to pack the gauge field and the Higgs field inside the horizon radius, which is smaller than the monopole radius $\ell_{A}$.

This thermodynamical stability indicates the possibility of a phase transition between two BHs (the monopole $\mathrm{BH}$ and the RN BH). As we show in Fig. 23, the entropy of the monopole $\mathrm{BH}$ with $\bar{\lambda}=1$ increases monotonically and connects with the RN BH branch at the junction point. If matter accretes onto the monopole $\mathrm{BH}$ and the mass increases to the junction point, we expect a second order phase transition from the monopole $\mathrm{BH}$ to the $\mathrm{RN} \mathrm{BH}$. Conversely, when the $\mathrm{BH}$ mass decreases via the Hawking radiation of the $\mathrm{RN} \mathrm{BH}$, a second order phase transition may occur at the junction point.

The case of small $\bar{\lambda}$ is more interesting. From the $\tilde{M}-\tilde{r}_{H}$ relation in Fig. 24, we find three branches: one RN BH branch (the line $\mathrm{BD}$ ) and two monopole $\mathrm{BH}$ branches (the curves $\mathrm{AC}$ and $\mathrm{BC}$ ). Using the stability analysis [17] and the catastrophe theory $[10,11]$, we find that both the rightside $\mathrm{RN} B \mathrm{BH}$ branch of the junction point $\mathrm{B}(\mathrm{BD})$ and the $\mathrm{AC}$ monopole branch are locally stable. The $\mathrm{BC}$ monopole branch is locally unstable. There are two stable states and one unstable state for some mass range. We expect the first order phase transition via the catastrophe theory, as follows. First, we consider the case that matter accretes onto a stable monopole BH. Figure 24 shows how such a monopole BH evolves. With mass accretion, the monopole $\mathrm{BH}$ evolves along the stable branch $\mathrm{AC}$. When the mass reaches the point $\mathrm{C}, \mathrm{BH}$ jumps onto the point $\mathrm{C}^{\prime}$ on the $\mathrm{RN} \mathrm{BH}$ branch and the horizon area (the $\mathrm{BH}$ entropy) changes discontinuously. It is the first order phase transition. For a case where the $\mathrm{BH}$ mass decreases via the Hawking radiation, the stable RN BH evolves along the stable branch DB and it eventually reaches the point $\mathrm{B}$. Then it jumps onto the point $\mathrm{B}^{\prime}$ on the stable monopole BH branch.

This cusp structure (two monopole branches) vanishes if the coupling constant $\bar{\lambda}$ is larger than the critical value $\bar{\lambda}_{\operatorname{cr}(1)}(\tilde{v})$, which depends on the VEV $v$. Hence, the phase transition between the $\mathrm{RN} \mathrm{BH}$ and the monopole $\mathrm{BH}$ is either (1) the second order where $\bar{\lambda}>\bar{\lambda}_{\operatorname{cr}(1)}$ or (2) the first order where $\bar{\lambda}<\bar{\lambda}_{\text {cr }(1)}$.

The above story is true only when $\bar{\lambda}$ and $\tilde{v}$ are rather small $[0 \leq \bar{\lambda}<O(1)$ and $0 \leq \tilde{v} \lesssim 0.2]$.

For more general values of the coupling constant $\bar{\lambda}$ and the VEV $\tilde{v}$, we may find two other critical curves, 


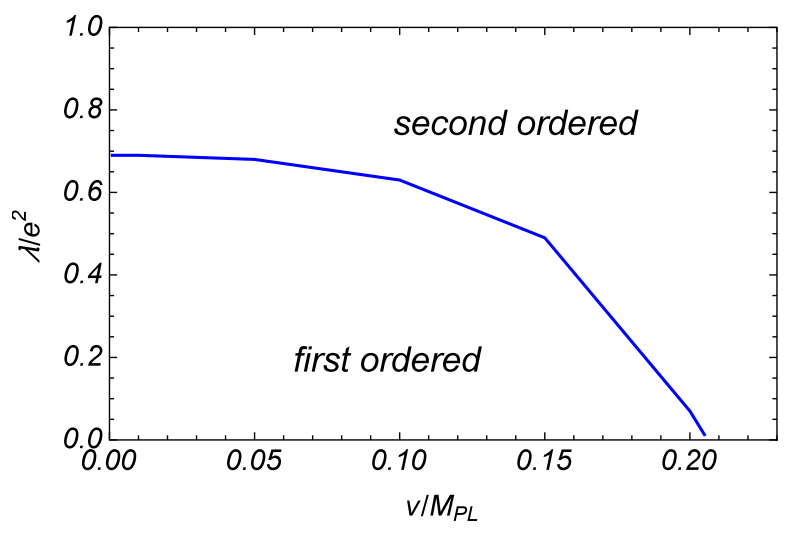

FIG. 26. The phase diagram of EYMH system with $\Lambda=0$. Below the blue curve $\bar{\lambda}=\bar{\lambda}_{\operatorname{cr}(1)}(\tilde{v})$, we find the first order phase transition. While above the critical curve, the second order phase transition is found.

$\bar{\lambda}=\bar{\lambda}_{\mathrm{cr}(2)}(\tilde{v})$ and $\bar{\lambda}=\bar{\lambda}_{\mathrm{b}}(\tilde{v})$. For $\bar{\lambda}_{\mathrm{cr}(1)}(\tilde{v})<\bar{\lambda}<\bar{\lambda}_{\mathrm{cr}(2)}(\tilde{v})$, we find the second order phase transition as we discussed above. In the range of $\bar{\lambda}_{\mathrm{cr}(2)}(\tilde{v})<\bar{\lambda}<\bar{\lambda}_{\mathrm{b}}(\tilde{v})$, we find a new type of first order phase transition. There exist the monopole $\mathrm{BH}$ branch as well as the $\mathrm{RN} \mathrm{BH}$ branch, but they are not connected. The monopole $\mathrm{BH}$ transits to the extreme $\mathrm{RN} \mathrm{BH}$, at which the horizon radius changes discontinuously. This is the first order phase transition.

Beyond $\bar{\lambda}_{\mathrm{b}}(\tilde{v})$, there is no monopole BH. For a given value of $\bar{\lambda}$, increasing $\tilde{v}$ further, we find the behavior of the metric function as shown in Fig. 25-i.e., the local minimum of $f(\tilde{r})$ appears-its value decreases and eventually vanishes at $\tilde{v}=\tilde{v}_{\mathrm{b}}(\bar{\lambda})$, beyond which there are no monopole $\mathrm{BH}$. The boundary curve $\tilde{v}=\tilde{v}_{\mathrm{b}}(\bar{\lambda})$ is equivalent to the curve $\bar{\lambda}=\bar{\lambda}_{\mathrm{b}}(\tilde{v})$.

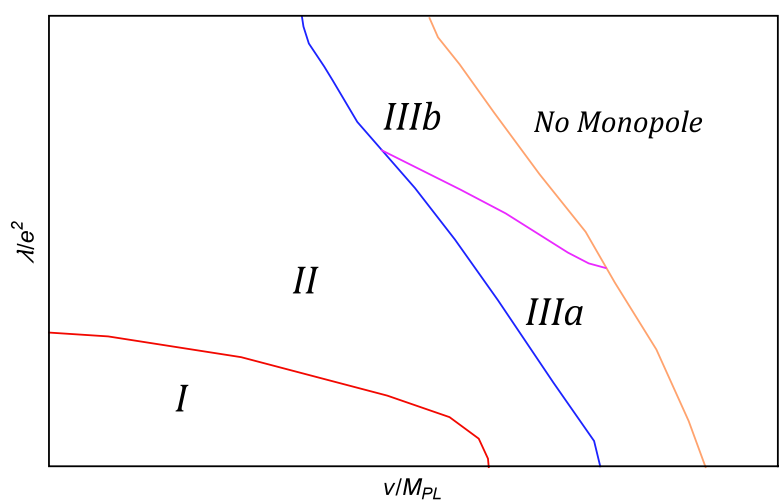

FIG. 27. The expected schematic phase diagram in the $\tilde{v}-\bar{\lambda}$ plane. Region I represents where the first order phase transition occurs, while region II shows where we find the second order phase transition. Regions IIIa and IIIb both denote where the "irreversible" phase transitions appear. In region IIIa, the phase transition from the monopole $\mathrm{BH}$ to the extreme $\mathrm{RN} \mathrm{BH}$ occurs, while, in region IIIb, we expect the transition from the monopole $\mathrm{BH}$ to the extreme monopole $\mathrm{BH}$.

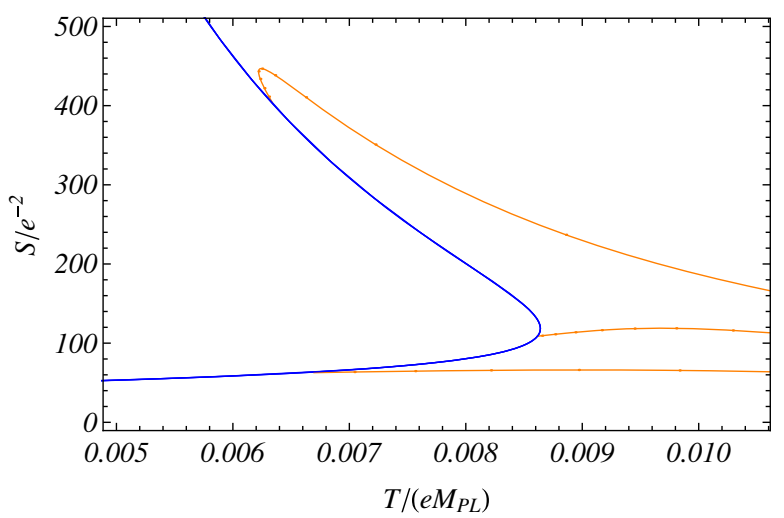

FIG. 28. $\quad \tilde{T}-\tilde{S}$ relation with a varying $\tilde{v}(\bar{\lambda}=0.1)$. The blue line represents a $\mathrm{RN} \mathrm{BH}$ and the orange lines represent a monopole $\mathrm{BH}$ with $\tilde{v}=0.05,0.1$, and 0.14 from the top.

For a very large value of $\bar{\lambda}$, we may find a similar first order phase transition, but with a different outcome, that is, the extreme monopole $\mathrm{BH}$ [15].

We show the phase diagram near the origin in Fig. 26 and summarize the expected schematic phase diagram in Fig. 27.

\section{b. $\mathrm{BH}$ in a heat bath}

Next, we consider a BH in a heat bath. In Fig. 28, we show the $\tilde{T}$ - $\tilde{S}$ relation for various values of $\tilde{v}$ fixing $\bar{\lambda}$, whose slope describes the heat capacity. If the slope is positive, the system in a heat bath is stable, while, if negative, it is unstable. For $\tilde{v}=0.05$, the entropy of the monopole $\mathrm{BH}$ changes its slope sign twice: negative $\rightarrow$ positive $\rightarrow$ negative. Hence, near the junction point to the $\mathrm{RH} \mathrm{BH}$ branch, the system is unstable, quickly becomes stable, then becomes unstable again. For $\tilde{v}=0.1$ and larger, the sign changes only once: positive $\rightarrow$ negative. As a result, the monopole $\mathrm{BH}$ in a heat bath is stable near the junction point, but it becomes unstable. For small values of $\tilde{v}$, a stable region is very small compared to the

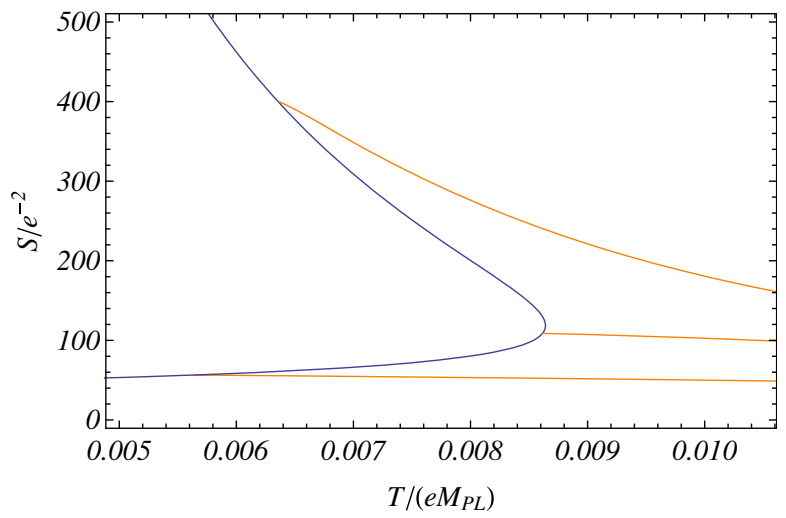

FIG. 29. $\tilde{T}-\tilde{S}$ relation with varying $\tilde{v}$ 's $(\bar{\lambda}=1)$. The blue line represents a $\mathrm{RN} \mathrm{BH}$ and the orange lines represent monopole BHs with $\tilde{v}=0.05,0.1$, and 0.15 from the top. 


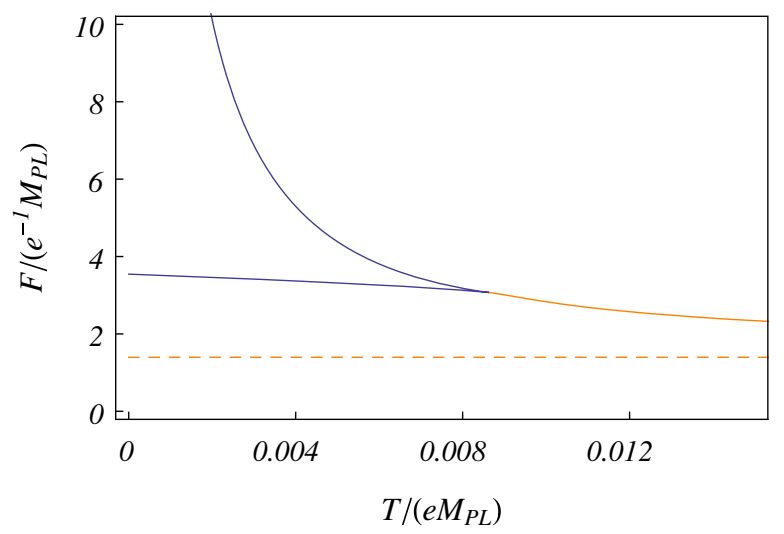

FIG. 30. $\tilde{T}-\tilde{F}$ relation $(\bar{\lambda}=0.1, \tilde{v}=0.1)$. The blue line represents a RN BH and the orange line represents a monopole $\mathrm{BH}$. The orange dashed line represents a gravitating monopole.

case with the large values of $\tilde{v}$. When $\bar{\lambda}$ is large, behaviors change significantly. In Fig. 29, we show the $\tilde{T}-\tilde{S}$ relation for $\bar{\lambda}=1$. In this case, the slope of the monopole $\mathrm{BH}$ branch is always negative, and there is no stable region. Only a RN BH with small entropy is stable.

The heat capacity gives an indicator of a thermodynamical stability of a system in a heat bath, but it is just a local stability. In order to discuss the global stability-i.e., which state is mostly preferred-we have to analyze the free energy. In Fig. 30, we present the Helmholtz free energy of the present system.

The RN BH branch starts from the extreme state with $T=0$ and turns backward at a critical temperature with an increase in the free energy. In the Einstein-Maxwell system, the first branch which includes the extreme state is globally stable. Above the critical temperature, no thermal spacetime with magnetic (or electric charge) is possible. In the present EYMH system, however, there are other branches of the monopole BH and the gravitating monopole. Since the free energy of the gravitating monopole is always smallest compared with those of the monopole $\mathrm{BH}$, as well as of the $\mathrm{RN} \mathrm{BH}$, the gravitating monopole in a heat bath is globally stable. Hence, there is no Hawking-Page transition in the EYMH system.

\section{APPENDIX B: HELMHOLZ FREE ENERGY}

In order to discuss the stability of a system in a heat bath, we calculate the Helmholtz free energy, which is obtained by the partition function defined by the path integral, ${ }^{4}$

\footnotetext{
${ }^{4}$ This is because we consider only a purely magnetically charged solution. If we discuss a purely electrically charged solution, the path integral gives the grand partition function [39].
}

$$
Z=\int \mathcal{D}[g, \phi] e^{-I_{E}[g, \phi]} .
$$

If we assume the main contribution in the integration comes from the classical solutions of the Euclidean action, the partition function is evaluated as $Z \approx \exp \left[-I_{E}^{\text {on-shell }}\right]$. Then the Helmholtz free energy $F$ is given by

$$
\beta F=I_{E}^{\text {on-shell }},
$$

where $\beta$ is the inverse temperature of the system and is identified with the Euclidean time period of the on-shell action. If we choose an arbitrary value of $\beta$ as an inverse temperature of the $\mathrm{BH}$ spacetime, the corresponding Euclidean BH spacetime may have a conical singularity at the "horizon." Since a spacetime with a conical singularity does not contribute to the partition function, the only allowed value of $1 / \beta$ must be the Hawking temperature $T$ given by Eq. (4.3).

On the other hand, in the case of a gravitating monopole, an arbitrary period of the Euclidean time $\beta$ is possible. We find a thermal gravitating soliton spacetime with an arbitrary temperature $T$.

Denoting a spacetime by $\mathcal{M}$ with the metric $g_{\mu \nu}$ and its boundary by $\partial \mathcal{M}$ with the induced metric $h_{m n}$, the action of the gravity system is given by

$$
I=I_{\mathrm{EH}}+I_{\mathrm{GHY}}+I_{\mathrm{C}}+I_{\text {matter }},
$$

where

$$
\begin{gathered}
I_{\mathrm{EH}}=\frac{1}{16 \pi G} \int_{\mathcal{M}} d^{4} x \sqrt{-g}(R-2 \Lambda) \\
I_{\mathrm{GHY}}=\frac{1}{8 \pi G} \int_{\partial \mathcal{M}} d^{3} x \sqrt{|h|} \varepsilon K \\
I_{\mathrm{C}}=\frac{1}{8 \pi G} \int_{\partial \mathcal{M}} d^{3} x \sqrt{|h|} \varepsilon K_{0},
\end{gathered}
$$

where $\varepsilon=g(\boldsymbol{n}, \boldsymbol{n})$, with $\boldsymbol{n}=n^{\mu} \partial_{\mu}$ being a normal vector of a hypersurface, $K=h^{a b} K_{a b}$ the trace of an extrinsic curvature $K_{a b}=\frac{1}{2}\left(\nabla_{\mu} n_{\nu}+\nabla_{\nu} n_{\mu}\right) e_{a}^{\mu} e_{b}^{\nu}$, and $K_{0}$ the counterterm to remove the divergence. Since the EinsteinHilbert term $I_{\mathrm{EH}}$ contains the second derivative of the metric, we have to introduce the Gibbons-Hawking-York term $I_{\mathrm{GHY}}$ in order to write the entire action only with the metric and the first derivative of the metric. The third term $I_{\mathrm{C}}$ is the so-called counterterm and is introduced to remove the divergence appearing in the Einstein-Hilbert term and the Gibbons-Hawking-York term.

In our setup, we take $K_{0}=-\frac{2}{L}\left(1+\frac{L^{2}}{4} R^{(3)}\right)$, where $R^{(3)}$ is the Ricci scalar on the boundary, which is given by $R^{(3)}=2 / r^{2}$ for a static and spherically symmetric spacetime, where $L \equiv \sqrt{-3 / \Lambda}$ is the AdS radius. 
Substituting our ansatz into the solution, the Euclideanized on-shell action $I_{E}^{\text {on-shell }}$ is written

$$
\begin{aligned}
I_{E}^{\text {on-shell }} & =I_{E, \mathrm{EH}}^{\text {on-shell }}+I_{E, \text { matter }}^{\text {on-shell }}+I_{E, \mathrm{GHY}}^{\text {on-shell }}+I_{E, \mathrm{C}}^{\text {on-shell }} \\
I_{E, \mathrm{EH}}^{\mathrm{on}-\mathrm{shell}}+I_{E, \text { matter }}^{\text {on-shell }} & =-4 \pi \int_{0}^{\beta} d t_{E} \int_{\text {bulk }} d r e^{-\delta} r^{2}\left[\frac{\Lambda}{8 \pi G}-f \frac{\left(w^{\prime}\right)^{2}}{e^{2} r^{2}}-\frac{1}{2} \frac{\left(w^{2}-1\right)^{2}}{e^{2} r^{4}}+\frac{\lambda}{4} v^{4}\left(h^{2}-1\right)^{2}\right], \\
I_{E, \mathrm{GHY}}^{\text {on-shell }} & =-\left.\frac{1}{2 G} \int_{0}^{\beta} d t_{E} e^{-\delta} r^{2}\left[\left(\frac{1}{2} f^{\prime}-f \delta^{\prime}\right)+\frac{2}{r} f\right]\right|_{r \rightarrow \infty}, \\
I_{E, \mathrm{C}}^{\text {on-shell }} & =\left.\frac{1}{2 G} \int_{0}^{\beta} d t_{E} e^{-\delta} r^{2} \sqrt{f} \frac{2}{L}\left(1+\frac{1}{2} \frac{L^{2}}{r^{2}}\right)\right|_{r \rightarrow \infty},
\end{aligned}
$$

where $t_{E}$ is the Euclidean time with the period of $\beta$.

The integration bulk region depends on whether the state is the $\mathrm{BH}$ solution or the soliton solution; in the case of the BH solution, the region is $\left[r_{H}, \infty\right)$, while it is $[0, \infty)$ for the soliton solution.

The Helmholtz free energy is the indicator of the most preferred state in a thermal bath. If there are multiple $\mathrm{BH}$ solutions with the same temperature, the minimum of the free energy chooses the most favored state in a thermal bath system. Such a state is globally stable in a thermal bath.

Once we know the solution of the EYMH system, we can evaluate $I_{E}^{\text {on-shell }}$ with Eq. (B7), then find the Helmholtz free energy $F$, which is given by Eq. (B2).
[1] B. Carter, Phys. Rev. Lett. 26, 331 (1971).

[2] R. Bartnik and J. McKinnon, Phys. Rev. Lett. 61, 141 (1988).

[3] P. Bizon, Phys. Rev. Lett. 64, 2844 (1990).

[4] S. Droz, M. Heusler, and N. Straumann, Phys. Lett. B 268, 371 (1991); M. Heusler, S. Droz, and N. Straumann, Phys. Lett. B 271, 61 (1991); P. Bizon and T. Chmaj, Phys. Lett. B 297, 55 (1992); T. Torii and K. Maeda, Phys. Rev. D 48, 1643 (1993).

[5] B. R. Greene, S. D. Mathur, and C. M. O’Neill, Phys. Rev. D 47, 2242 (1993).

[6] F. A. Bais and R. J. Russell, Phys. Rev. D 11, 2692 (1975).

[7] K. Lee, V. P. Nair, and E. J. Weinberg, Phys. Rev. D 45, 2751 (1992).

[8] M. E. Ortiz, Phys. Rev. D 45, R2586 (1992).

[9] P. Breitenlohner, P. Forgács, and D. Maison, Nucl. Phys. B383, 357 (1992).

[10] K. Maeda, T. Tachizawa, T. Torii, and T. Maki, Phys. Rev. Lett. 72, 450 (1994).

[11] T. Tachizawa, K. Maeda, and T. Torii, Phys. Rev. D 51, 4054 (1995).

[12] P. Breitenlohner, P. Forgács, and D. Maison, Nucl. Phys. B442, 126 (1995).

[13] A. Lue and E. J. Weinberg, Phys. Rev. D 60, 084025 (1999).

[14] A. Lue and E. J. Weinberg, Phys. Rev. D 61, 124003 (2000).

[15] Y. Brihaye, B. Hartmann, and J. Kunz, Phys. Rev. D 62, 044008 (2000).

[16] A. Lue and E. J. Weinberg, Gen. Relativ. Gravit. 32, 2113 (2000).

[17] P. C. Aichelburg and P. Bizon, Phys. Rev. D 48, 607 (1993).
[18] R. Thom, Structural Stability and Morphogenesis (Benjamin, New York, 1975).

[19] J. M. Bardeen, B. Carter, and S. W. Hawking, Commun. Math. Phys. 31, 161 (1973).

[20] S. W. Hawking, Commun. Math. Phys. 43, 199 (1975).

[21] J. M. Maldacena, Int. J. Theor. Phys. 38, 1113 (1999); Adv. Theor. Math. Phys. 2, 231 (1998).

[22] See, for example, R. Emparan, J. High Energy Phys. 06 (1999) 036; A. Chamblin, R. Emparan, C. V. Johnson, and R. C. Myers, Phys. Rev. D 60, 064018 (1999); S. S. Gubser, Phys. Rev. D 78, 065034 (2008); Phys. Rev. Lett. 101, 191601 (2008).

[23] E. Winstanley, Classical Quantum Gravity 16, 1963 (1999).

[24] J. Bjoraker and Y. Hosotani, Phys. Rev. Lett. 84, 1853 (2000).

[25] R. B. Mann, E. Radu, and D. H. Tchrakian, Phys. Rev. D 74, 064015 (2006).

[26] O. Kichakova, J. Kunz, E. Radu, and Y. Shnir, Phys. Lett. B 747, 205 (2015).

[27] A. R. Lugo and F. A. Schaposnik, Phys. Lett. B 467, 43 (1999).

[28] A. R. Lugo, E. F. Moreno, and F. A. Schaposnik, Phys. Lett. B 473, 35 (2000).

[29] O. Kichakova, J. Kunz, E. Radu, and Y. Shnir, Phys. Rev. D 86, 104065 (2012).

[30] A. R. Lugo, E. F. Moreno, and F. A. Schaposnik, J. High Energy Phys. 03 (2010) 013.

[31] D. Allahbakhshi and F. Ardalan, J. High Energy Phys. 10 (2010) 114. 
[32] A. R. Lugo, E. F. Moreno, and F. A. Schaposnik, J. High Energy Phys. 11 (2010) 081.

[33] S. Bolognesi and D. Tong, J. High Energy Phys. 01 (2011) 153.

[34] S. Bolognesi, Nucl. Phys. B845, 324 (2011).

[35] P. Sutcliffe, J. High Energy Phys. 08 (2011) 032.

[36] D. Allahbakhshi, J. High Energy Phys. 09 (2011) 085.
[37] G. L. Giordano and A. R. Lugo, J. High Energy Phys. 07 (2015) 172.

[38] E. Radu and D. H. Tchrakian, Phys. Rev. D 71, 125013 (2005).

[39] S. R. Coleman, J. Preskill, and F. Wilczek, Nucl. Phys. B378, 175 (1992). 